Eechnical Note

\title{
LECTURES ON ION-ATOM COLLISIONS
}

M. R. C. MCDOWELL

U. S. DEPARTMENT OF COMMERCE NATIONAL BUREAU OF STANDARDS 


\section{THE NATIONAL BUREAU OF STANDARDS}

\section{Functions and Activities}

The functions of the National Bureau of Standards are set forth in the Act of Congress, March 3, 1901, as amended by Congress in Public Law 619, 1950. These include the development and maintenance of the national standards of measurement and the provision of means and methods for making measurements consistent with these standards; the determination of physical constants and properties of materials; the development of methods and instruments for testing materials, devices, and structures; advisory services to government agencies on scientific and technical problems; invention and development of devices to serve special needs of the Government; and the development of standard practices, codes, and specifications. The work includes basic and applied research, development, engineering, instrumentation, testing, evaluation, calibration services, and various consultation and information services. Research projects are also performed for other government agencies when the work relates to and supplements the basic program of the Bureau or when the Bureau's unique competence is required. The scope of activities is suggested by the listing of divisions and sections on the inside of the back cover.

\section{Publications}

The results of the Bureau's research are published either in the Bureau's own series of publications or in the journals of professional and scientific societies. The Bureau publishes three periodicals available from the Government Printing Office: The Journal of Research, published in four separate sections, presents complete scientific and technical papers; the Technical News Bulletin presents summary and preliminary reports on work in progress; and the Central Radio Propagation Laboratory Ionospheric Predictions provides data for determining the best frequencies to use for radio communications throughout the world. There are also five series of non periodical publications: Monographs, Applied Mathematics Series, Handbooks, Miscellaneous Publications, and Technical Notes.

A complete listing of the Bureau's publications can be found in National Bureau of Standards Circular 460, Publications of the National Bureau of Standards, 1901 to June 1947 (\$1.25), and the Supplement to National Bureau of Standards Circular 460, July 1947 to June 1957 (\$1.50), and Miscellaneous Publication 240, July 1957 to June 1960 (includes Titles of Papers Published in Outside Journals 1950 to 1959) (\$2.25); available from the Superintendent of Documents, Government Printing Office, Washington 25, D.C. 


\title{
NATIONAL BUREAU OF STANDARDS
}

\author{
Eechnical Note 185
}

ISSUED MARCH 15, 1963

\section{LECTURES ON ION-ATOM COLLISIONS}

\author{
M. R. C. McDowell \\ Royal Holloway College \\ University of London
}

Lectures delivered at the Joint Institute for Laboratory Astrophysics, University of Colorado, during September, 1962.

NBS Technical Notes are designed to supplement the Bureau's regular publications program. They provide a means for making available scientific data that are of transient or limited interest. Technical Notes may be listed or referred to in the open literature. 

LECTURES ON ION-ATOM COLLISIONS

By

M. R. C. McDowe $11 *$

PREFACE

This report contains lectures delivered at the Joint Institute for Laboratory Astrophysics, University of Colorado, during September, 1962. The material of the first four is largely taken from recent books by D. R. Bates, and was presented for pedagogic purposes only. It does not purport, in any sense, to be a record of original work by the author. Any new material is confined to the last two lectures. The numerical results quoted are taken, in part, from as yet unpublished work by M. B. McElroy (Belfast), Rudd and Jorgens on (University of Nebraska), and the author. Reference to this work should be made to the appropriate publication.

The author takes this opportunity of thanking the Bureau of Standards and the University of Colorado for their hospitality during this sumer.

* Permanent address: Roya1 Holloway College, University of London 


\section{Contents}

Page

Lecture I. The Impact Parameter Formulation of the Theory of Atom-Atom Collisions . . . . . . . . . . 1

Lecture II. Applications of the First Born Approximation. . . 12

Lecture III. Slow Charge Transfer. . . . . . . . . . 22

Lecture IV. Charge Transfer in Other Cases. . . . . . . . 34

Lecture V. (i) Further Remarks on Charge Transfer

(ii) Curve - Crossing . . . . . . . . . 44

Lecture VI. Ionization of Atomic Systems by Fast Protons. . . 56 


\section{LECTURE I}

\section{The Impact Parameter Formulation of The Theory of Atom-Atom Collisions}

We will describe in this lecture the treatment of atom-atom (including ion-atom collisions) developed by Bates and his coworkers $(1-3)$ paying particular attention to the sequence of Born approximations and to the so called "distortion" approximation, but will leave to a later lecture any specific treatment of rearrangement collisions.

The theory to be described makes two basic assumptions.

(i) The relative motion of the colliding systems is sufficiently fast that the usual Born-Oppenheimer separation of electronic and nuclear motion is valid, and further, that the nuclear orbits are rectilinear, the velocity of relative motion $\underline{v}$ being constant throughout the collision.

(ii) The velocity of relative motion $|\underline{v}|$ is sufficiently low that nuclear and relativistic effects may be ignored. Assumption (i) (a), the Born-Oppenheimer separation, is valid at all energies of interest if both the systems are in $S$ states, but may lead to serious errors at thermal velocities in other cases. Assumption (ib), is equivalent to supposing that the de Broglie wave length $\lambda$ of the incident particle, in the system in which the target is at rest, is small compared with atomic dimensions.

That is

$$
\lambda=\frac{\hbar}{m_{i} v} \ll a_{0}
$$


For incident protons this is satisfied if $\mathrm{E}_{\mathrm{i}}<10 \mathrm{ev}$, and for heavier atoms at correspondingly higher energies. However, even for atoms as heavy as Mercury, $\lambda$ is $\sim 0.1$ a at $300^{\circ} \mathrm{K}$, so one should not suppose that cross sections calculated by the theory to be described are necessarily adequate at thermal energies, or that estimates of rate coefficients so obtained are applicable to upper atmospheric conditions.

Assumption (ii) is valid, insofar as relativistic effects are concerned, for heavy particle impact, up to several tens of Mev, provided inner shell electrons are ignored; but modification of the cross sections by the nuclear forces becomes important when $\lambda \sim 10^{-12} \mathrm{~cm}$. (If much of the contribution comes from small impact parameters), which is $10 \mathrm{Mev}$ for $\mathrm{H}^{+}$impact, but correspondingly lower for heavier atoms. For example, $\lambda\left(\mathrm{A}^{+}\right)$is $10^{-12} \mathrm{~cm}$ at $100 \mathrm{kev}$. We can suppose that the limits of applicability of the theory are approximately

$$
10 \mathrm{ev}<\mathrm{E}_{1}<10 \mathrm{Mev}
$$

for $\mathrm{H}^{+}$impact.

We consider an unperturbed system, Hamiltonian $\mathrm{H}_{\mathrm{O}}$, in an eigenstate $\Phi_{S}$ supposed known, with energy $\epsilon_{S}$

$$
H_{0} \Phi_{s}=\epsilon_{s} \Phi_{s}
$$

and suppose that the effect of the collision is to introduce a perturbation $V$, which is an implicit function of the time. Then the state of the system at time $t$ may be described by a suitable 
solution of

$$
\left(H-i \frac{\partial}{\partial t}\right) \Psi_{n}=0
$$

where

$$
H=H_{0}+V
$$

It is customary to expand $\Psi_{n}$ in terms of the $\phi_{S}$ (all S)

$$
\Psi_{n}=\int_{s} a_{n s}(t) \phi_{s} e^{-i \varepsilon_{s} t}
$$

where $a_{n s}(t)$ are the occupation numbers of state $s$ at time $t$, the system being originally in state $\mathrm{n}$, and the collision taking place at impact parameter $p$. In particular one supposes the target to be at rest at the origin of co-ordinates in a frame $0 x y z$, and that the projectile moves with velocity $\underline{\mathrm{v}}$ parallel to and at a distance $p$ from the $z$ axis, the trace of $i t s$ path in the $x y$ plane at $P$ being such that $O P$ makes an angle $\Phi$ with the $X$ axis. (Fig. I).

Then

$$
X=p \cos \Phi ; Y=p \sin \Phi ; z=v t
$$

where $Z(p)=0$.

\section{FIGURE I.}

(See Separate Sheet) 
4

The probability that such a passage causes a transition from state $n$ to state $m$ is clearly $\left|a_{n m}(+\infty)\right|^{2}$ and integrating over all impact parameters (supposing $V$ independent of $\Phi$ ) the cross section is

$$
Q_{n m}(E)=2 \pi \int_{0}^{\infty}\left|a_{n m}(+\infty)\right|^{2} p d p
$$

Our aim, therefore, is to obtain an expression for $\left|a_{n m}(\infty)\right|^{2}$, and to evaluate it, for the particular transition of interest.

Let us substitute $(1.5)$ in (1.3), making use of (1.2) to

obtain

$$
v \int_{s} a_{n s}(t) \phi_{s} e^{-i \varepsilon_{s} t}=i \int_{s} \dot{a}_{n s}(t) \phi_{s} e^{-i \varepsilon_{s} t}
$$

and multiplying on the left by $\phi_{\mathrm{m}^{*}} i \epsilon_{\mathrm{m}} \mathrm{t}$, integrate over all the electronic coordinates. We obtain

$$
\int_{s} a_{m s}(t) v_{m s} e^{i \Delta \varepsilon_{m s} t}=i \dot{a}_{n m}(t)
$$

where

$$
\begin{aligned}
v_{m s} & =\int \phi_{m}^{*} v \phi_{s} d \underline{r} \\
\diamond \quad \Delta \varepsilon_{m s} & =\varepsilon_{m}-\varepsilon_{s}
\end{aligned}
$$

This is a set of coupled integro-differential equn's, and does not admit of a general $\operatorname{sol}^{\mathrm{n}}$.

Let us suppose

(i) $V_{n j}=0 ; j \neq n$ or $m$ and impose the boundary conditions

$(1.10)$

(ii) $a_{n n}(-\infty)=1 ; a_{n m}(-\infty)=0, m \neq n$. 
In virtue of (i), (1.8) reduces to

$$
i a_{n m}(t)=a_{n n}(t) V_{m n} e^{i \Delta \varepsilon_{m n} t}
$$

If $m=n$, we may readily integrate this and obtain

$$
a_{n n}(z)=1-\frac{i}{v} \int_{-\infty}^{z} v_{n n} d z^{\prime}
$$

where we have changed the variable for convenience. If now $V_{n n}$ is small for all $\mathrm{z}$, or if $\mathrm{v}$ is very large, we may take $a_{n n} \doteqdot 1$ (all z), and integrate (1.11) for $\mathrm{n} \neq \mathrm{m}$ to obtain

$$
a_{n m}(z)=-\frac{i}{v} \int_{-\infty}^{z} v_{m n} e^{i \Delta \varepsilon_{m n} z^{\prime} / v} d z^{\prime}
$$

Thus, to this approximation, the cross section is

$$
Q_{n m}=2 \bar{u} \int_{0}^{\infty} \frac{1}{v^{2}}\left|\int_{-\infty}^{+\infty} v_{m n} \exp \left\{i \frac{\Delta \varepsilon_{m n} z^{\prime}}{v}\right\} d z^{\prime}\right|^{2} p d p
$$

and is referred to as the 1 st Born approximation since the assumptions made are mathematically equivalent to the usual wave formulation of the first Born approximation, when the nucleii are assumed to move as classical particles. A rigorous proof of the equivalence is provided by Frame ( 7 ).

This approximation is justified only if the sum of the transition probabilities remains small throughout the encounter

$$
\text { ie } \quad \sum_{p}\left|a_{u p}(z)\right|^{2} \ll 1
$$


6

If this is not the case, improvements can be made in two directions.

We can take specific account of those $a_{n p}$ judged to be most important, to a high order of approximation ("Distortion approximatron"), or partial account of all of them (at least in principle) by a "Ind Born Approximation". In the later, we start from (1.8) and use (1.12) and (1.13) on the R.H.S. This yields

$$
\begin{aligned}
i v \frac{\partial a_{n m}}{\partial z}= & -\frac{i}{v} \int_{s \neq u} v_{m s} e^{i \varepsilon_{m s} z / v} \int_{-\infty}^{z} v_{u s} e^{i \varepsilon_{n s} z^{\prime} / v} d z^{\prime} \\
& +\left[1-\frac{i}{v} \int_{-\infty}^{z} v_{u n} d z^{\prime}\right] v_{n m} e^{i \varepsilon_{m n} z / v}
\end{aligned}
$$

which may be written as

$$
i v \frac{\partial a_{m n}}{\partial z}=\left(v_{n m}+w_{n m}\right) e^{i \varepsilon_{m n} z / v}
$$

where

$$
W_{n m}=-\frac{i}{v} \int_{s} v_{m s} e^{i \varepsilon_{m s} z / v} \int_{-\infty}^{z} v_{u s} e^{i \varepsilon_{u s} z^{\prime} / v} d z^{\prime}
$$

As $v \rightarrow \infty$, the exponential can be replaced by unity and

$$
W_{n m} \doteq-\frac{i}{v} \int_{s} v_{m s} v_{u s} \doteq-\frac{1}{v}\left(v^{2}\right)_{n m}
$$

which is of the second order in the interactions.

If we integrate $(1.17)$, we have

$$
a_{n m}(z)=\frac{i}{i v} \int_{-\infty}^{z}\left(v_{m n}+w_{m n}\right) e^{i \varepsilon_{m n} z^{\prime} / v} d z^{\prime}
$$


giving for the cross section

$$
Q_{n m}=\frac{2 \pi}{v^{2}} \int_{0}^{\infty}\left|\int_{-\infty}^{+\infty}\left(v_{m n}+w_{m n}\right) e^{i \varepsilon_{m n} z / v} d z\right|_{4}^{2} p d p(1.20)
$$

where the subscript 4 indicates that all terms of the 4 th order in the interactions should be dropped, since further terms of this order arise from products of 3 rd order and 1 st order terms and these have not been included. (8) In principle (1.20) takes proper account, to the third order in the interactions, of all the matrix elements, but in practice $\mathrm{W}_{\mathrm{mn}}$ is approximated by a sum of what are considered the two or three most important matrix elements, including say $V_{m m}$ and $V_{m n}$ and terms differing from these only in magnetic quantum number.

The distortion approximation, on the other hand, begins by noting that of all the terms in (1.8), the only one whose magnitude is not affected by oscillatory factors is that involving $V_{\operatorname{mm}}$, the so-called "secular" term. The distortion approximation takes this term almost completely into account.

We first of all remove the secular terms by writing

$$
C_{n s}=a_{n s} \exp \left\{i \int_{0}^{t} V_{s s} d t^{\prime}\right\}
$$

so that (1.8) is replaced by

$$
i \dot{C}_{n m}=\int_{s \neq m} C_{n s} V_{m s} \exp \left\{-i \int_{0}^{t} \gamma_{m s} d t^{\prime}\right\}
$$


8

with

$$
\gamma_{m s}=\left(\varepsilon_{m}+v_{m m}\right)-\left(\varepsilon_{s}+v_{s s}\right)
$$

so that the resultant formulae are analagous to those of the 1 st Born approximation, except that the unperturbed eigenenergies $\epsilon_{S}$ are replaced by the 1 st order perturbed eigenenergies.

$$
\eta_{s}=\varepsilon_{s}+v_{s s}
$$

Higher approximations may be obtained by retaining more than two terms in the initial expansions.

A very simple alternative derivation of (1.22) has been given by Bates (9). We start from (1.8) and the pst Born solution for $a_{n}$. We have

$$
\frac{\partial a_{n}}{\partial z}=\frac{1}{v} \int_{s} a_{s}(z) v_{n s} e^{-i \varepsilon_{s n} z}
$$

and suppose $a_{s}=o(s \neq n)$ to obtain

$$
\frac{\partial a_{n}}{\partial z}=\frac{1}{v} a_{n} v_{n n}
$$

which integrates to give

$$
a_{n}(z)=\exp \left\{-\frac{i}{v} \int_{0}^{z} v_{n u} d z^{\prime}\right\}
$$

Now to obtain $a_{m}(z)$ we retain only $a_{m}$ and $a_{n}$, so that $\left(1.8^{1}\right)$ becomes

$i \frac{\partial Q_{m}}{\partial z}=\frac{1}{v}\left\{a_{n}(z) V_{n m}+a_{m}(z) V_{m m}\right\}$ 
9

so using $(1.26)$,

$$
\frac{i}{\partial a_{m}}=\frac{i}{v}\left[a_{m} v_{m m}+v_{u m} \exp \left\{-\frac{i}{v} \int_{0}^{z} v_{n n} d z^{\prime}\right\}\right]
$$

which may readily be integrated to yield

$$
a_{m}(+\infty)=-\frac{i}{v} \int_{-\infty}^{+\infty} V_{u m} \exp \left\{-\frac{i}{v} \varepsilon_{u m} z-i \beta_{n m}\right\} d z
$$

where

$$
\beta_{n m}=\frac{i}{v} \int_{0}^{z}\left(v_{u n}-v_{m m}\right) d z^{\prime}
$$

Thus the cross section can be written

$$
Q_{u m}=\left.\frac{2 \pi}{v^{2}} \int_{\text {with } \gamma_{m n} \text { given by }}^{\infty} \int_{-\infty}^{+\infty} v_{n m} e^{-i \gamma_{m n} z / v} d z\right|^{2} p d p .
$$




\section{REFERENCES}

1. Bates, D. R., Quantum Theory (Ed. Bates), Ch. 8 [N. Y., Academic Press] (1961).

2. Bates, D. R., "Atomic and Molecular Processes" (Ed. Bates), Ch. 14, [N. Y. Academic Press], (1962).

3. Bates, D. R. and McCarro1, R., Adv. Phys. 11, 1. (1962).

4. Wu, T. Y. and Bhatia, A., J. Chem. Phys., 24, 48. (1956).

5. Dalgarno, A., and McCarrol, R., Proc.Roy. Sco., A, 237, 383 (1956).

6. Dalgarno, A. and McCarrol, R., Proc. Roy. Soc. A, 239, 413 (1957).

7. Frame, W. A., P. Comb. Phil Soc. 27, 511., (1931).

8. Moisiewitsch, B. L., Atomic and Molecular Processes (1oc. cit.), Ch. 9, (1962).

9. Bates, D. R., Proc. Phys. Soc. 73, 227. (1959). 
FIG . 1

Coordinates used in the impact parameter formulation.

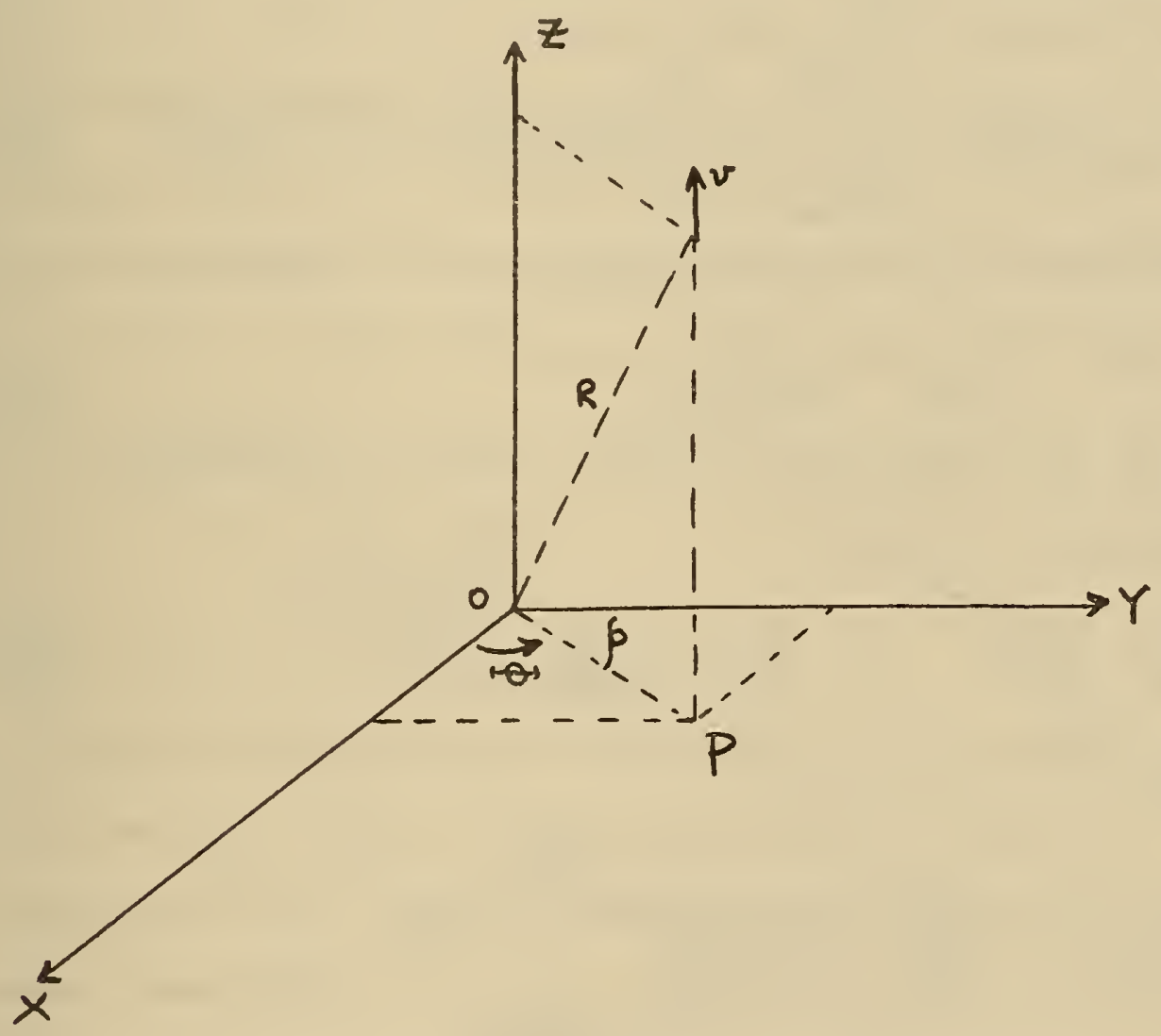


12

LECTURE II

Applications of The First Born Approximation

§ (1). The 1S-2S Excitation of $\mathrm{H}$ by $\mathrm{H}^{+}$: Impact Parameter Form

The cross section is given by

$$
Q_{1 s-2 s}(v)=2 \pi \int_{0}^{\infty}\left|a_{1 s, 2 s}(+\infty)\right|^{2} p d p \text {, units } a_{0}^{2}
$$

where we have seen (1.13) that

$$
a_{15,2 s}(+\infty)=-\frac{i}{v} \int_{-\infty}^{+\infty} V_{15,2 s} \exp \left\{i \Delta \varepsilon_{1 s, 2 s} z^{\prime} / v\right\} d z^{\prime}
$$

Following Bates $(1)$ we have that the interaction potential is

$$
H^{\prime}=\frac{1}{R}-\frac{1}{\mid \underline{\underline{x}-\underline{R} \mid}}
$$

where $\underline{\mathrm{R}}$ is the internuclear separation, and $\underline{\underline{r}}$ the electron's position vector. Taking

$$
\phi_{15}=\frac{1}{\sqrt{\pi}} e^{-r} ; \quad \phi_{2 s}=\frac{1}{4 \sqrt{\alpha \bar{u}}}(\alpha-r) e^{-r / 2}
$$

we have

$$
\begin{aligned}
V_{15,25} & =-\frac{\sqrt{2}}{8 \bar{u}} \int \frac{(2-r)}{|r-\underline{R}|} e^{-3 r / 2} d r \\
& =-\frac{2 \sqrt{2}}{27}(2+3 R) c^{-3 R / 2}
\end{aligned}
$$

Using this in (2.2) and noting that it is an even function of $Z$, we have (1)

$$
a_{15,2 s}=-\frac{2^{5 / 2}}{2 y v} \int_{0}^{\infty}(2+3 R) e^{-3 R / 2} \cdot \cos \left(\frac{3 z^{\prime}}{8 v}\right) d z^{\prime}
$$


which is the sum of two standard Founer cosine transforms, and is equal to

$a_{15,2 s}=\frac{2^{13 / 2} p^{2} v}{9\left(1+16 v^{2}\right)} K_{2}\left\{\frac{3 p}{8 v}\left(1+16 v^{2}\right)^{1 / 2}\right\}$

where $K_{2}(x)$ is the 2 nd order moditied Bessel function of the first kind, and the cross section may be readily evaluated numerically. The results are shown in Fig. (2.1). It may readily be seen that when $v$ is large $\cos \left(\frac{3 z^{\prime}}{8 v}\right) \sim 1$, so that the cross section is proportional to $\mathrm{v}^{-2}$. Further, when $\mathrm{v}$ is small the rapid oscillations of the cosine factor make the cross section go rapidly to zero with $v$. If we take $v_{1 s \text {, as }}$ to have range $\alpha$, then $\left|a_{1 s, a s}\right|^{2}$ is a maximum when

$$
v \doteq \frac{\varepsilon_{15,25} \alpha}{\lambda}
$$

which is the usual adiabatic criteria.

Similar calculations have been carried out for transitions to the $2 \mathrm{p}_{0}$ and $2 \mathrm{p} \pm$ l states, and for these transitions when the incident particle is a hydrogen atom in the ground state, which remains unexcited $(2)$ The cross sections obtained are identical to these obtained earlier (3) by the usual wave formulation. The collected results have been presented by Bates $(4)$. However, in the impact parameter formulation, the values of the transition probabilities allow one to make some estimate of their sum $\Sigma^{\prime}\left|a_{n p}\right|^{2}$ at impact parameter $p$, and yield some information on the range of 
14

validity of the Born approximation as a function of impact

parameter. Since most of the contribution comes from small impact

parameter. Bates (4) has evaluated

$$
p^{\prime}=\lim _{p \rightarrow 0} \sum_{s}^{\prime}\left|a_{w s}\right|^{2}
$$

and considers the criterion $P^{\prime}<1$ satisfied if $P^{\prime}<0.15$, but grossly violated if $\mathrm{P}^{\prime}>0.5$. For $\left[\mathrm{H}^{+}, \mathrm{H}(1 \mathrm{~S})\right]$ collisions he finds the Born approximation satisfactory (provided distortion is also unimportant) for $E_{i}>200 \mathrm{kev}$, and completely unreliable for $E_{i}<50$ nev.

§(2). The Wave Formulation

Following Mott and Massey (5) we have

$$
Q_{n m}(v)=\frac{1}{2 \bar{n} v_{n}^{2}} \int_{K_{\min }}^{k_{\max }}\left|N_{n m}\right|^{2} k d k
$$

where

$$
\underline{K}=\underline{k}_{f}-\underline{k_{i}}
$$

and we may take

$$
K_{\text {min }} \doteq \frac{\varepsilon_{n m}}{v_{n}}\left\{1+\frac{\varepsilon_{n r n}}{2 \mu v^{2}}+\ldots .\right\}
$$

and $\mathrm{K}_{\max }$ as infinite. The interaction potential and the wave functions are identical to those given in $\$ 1$. Substituting, and making use of Bethe's integral

$$
\int \frac{e^{i \underline{R} \cdot(r-\underline{R})}}{|\underline{r}-\underline{R}|} d \underline{r}=\frac{4 \bar{n}}{R^{2}} e
$$

$i k \cdot S$ 
one obtains

$$
Q_{n m}(v)=\frac{8}{S_{i}^{2}} \int_{k_{\min }}^{\infty}\left|I_{n m}\right|^{2} K^{-3} d k \quad\left(\bar{n} a_{0}^{2}\right)
$$

where, in

$$
Q_{n m}(v), I_{n m}=\int X_{n}^{*}(r) x_{m}(r) e^{-i \underline{k} \cdot r} d r
$$

and $X_{n}, X_{m}$ are the initial and final atomic orbitals. For the $1 \mathrm{~s} \rightarrow$ 2s transition this becomes,

$$
\begin{aligned}
I_{n m} & =\frac{\sqrt{2}}{8 \pi} \int(2-r) e^{-3 r / 2} e^{-i k \cdot R} d r \\
& =\frac{\sqrt{2}}{2 k} \int_{0}^{\infty}(2-r) r e^{-3 r / 2} \sin (k r) d r
\end{aligned}
$$

which is readily evaluated by a suitable sequence of differentiations of

$$
\int_{0}^{\infty} e^{-\alpha r} \cos (\beta r)=\frac{1}{\left(\alpha^{2}+\beta^{2}\right)} \cdot\left\{\begin{array}{l}
\alpha \\
\beta
\end{array}\right.
$$

to yield

$$
I_{15,2 s}=\frac{2^{17 / 2} k^{2}}{\left(4 k^{2}+9\right)^{3}}
$$

and the cross section may then be evaluated from (2.13) by numerical integration. The method may readily be generalized to collisions of a bare ion of charge $z_{b}$ incident on an ion isoelectronic with $\mathrm{H}$ of charge $\mathrm{z}_{\mathrm{a}}$. 
The resultant expression reduces( ${ }^{(3)}$ at high energies to

$$
\left.\begin{array}{l}
\left.Q_{15,25}\left(E_{i}\right)=\frac{11}{x} \frac{Z_{b}^{2}}{z_{a}^{4}}\left(1-\frac{7 \cdot 8}{x}\right) \quad \overline{n a_{0}^{2}}\right\} \\
\text { where } x=E_{i} / M_{b} z_{a}^{2}
\end{array}\right\}
$$

§(3). If the incident projectile is not a bare ion but another atomic system, a similar analysis shows that $I_{n m}$ is replaced by a product of two factors, one being a matrix element for transitions of the target and one of the incident atom, these factors being identical to those in the single excitation case.

For

$$
H(1 s)+H(15) \rightarrow H(n l)+H\left(n^{\prime} l^{\prime}\right)
$$

$$
Q_{\left(\text {sinle, } 1 s \rightarrow n^{\prime} l^{\prime}\right)}=\frac{8}{s i^{2}} \int_{k_{m m}}^{\infty}\left|I(l s \rightarrow n l) I\left(1 s \rightarrow u^{\prime} l^{\prime}\right)\right|^{2} k^{-3} d k
$$

At sufficiently high energies $\mathrm{K}_{\min } \rightarrow 0$, and sum rules may be applied to the matrix elements, to deduce the total cross section for all inelastic collisions ${ }^{(3)}$ ( $E_{i}$ in kev.),

$$
Q(1 s, 1 s, \rightarrow \Sigma, \Sigma) \doteq \frac{2 i n}{E_{i}} \quad\left(\bar{n} a_{0}{ }^{2}\right)
$$

where the major contribution comes from double ionization processes. Some representative results are shown in Fig. (2.2), and a table of the transitions for which results are available follows: 


\section{REFERENCES}

1. Bates, D. R., Quantum Theory (loc. cit.), (1961).

2. Bates, D. R., Proc. Roy. Soc. A, 425, 299, (1958).

3. Bates, D. R. and Griffing, G. W., (1953), Proc. Phys.

Soc. A 66, 961, (1954), Proc. Phys. Soc. A 67, 663.

4. Bates, D. R., Atomic and Molecular Processes (loc. cit.), (1962).

5. Mott, N. F. and Massey, H. S. W., Theory of Atomic Collisions, 2nd Ed. (0.T.P.), (1948). 
FIG. 2.1. (From Bates 1961)

\section{BORN 2 DISTORTION APPROXIMATIONS TO CROSS SECTIONS FOR}

$H(1 s)+H^{+} \longrightarrow H(2 s)+H^{+}$

BATES, 1961

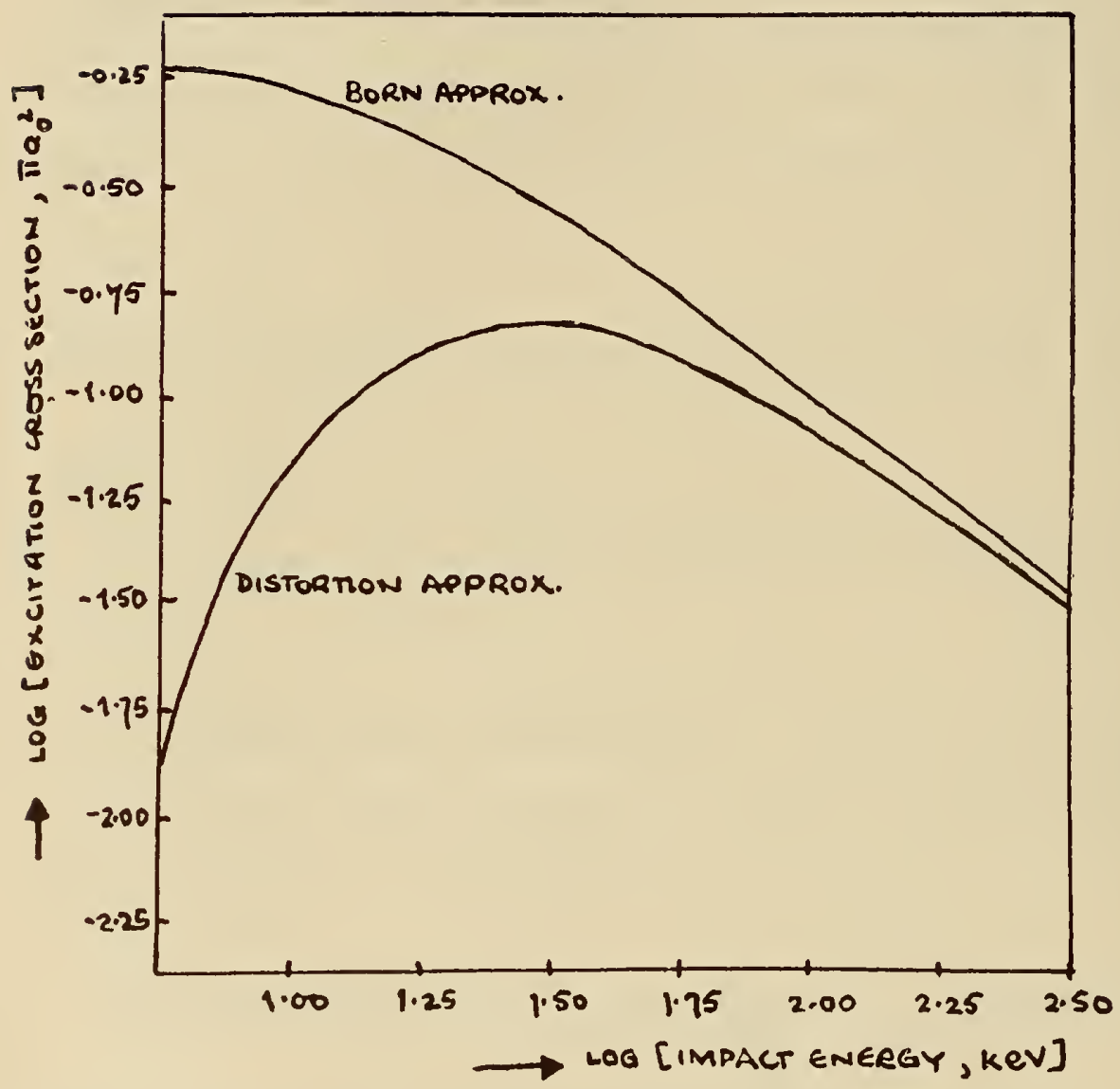


FIG. 2.2

FIRST BORN APPROXIMATION TO THE CROSS SECTLN. IMPACT ENERGY WURES FOR:-

$$
H(15)+H(15) \rightarrow H(2 s)+H(n e)
$$

WHERE ne REPRESENTS $2 s, 2 p, 3 s, 3 p, 3 d$ or $C$.

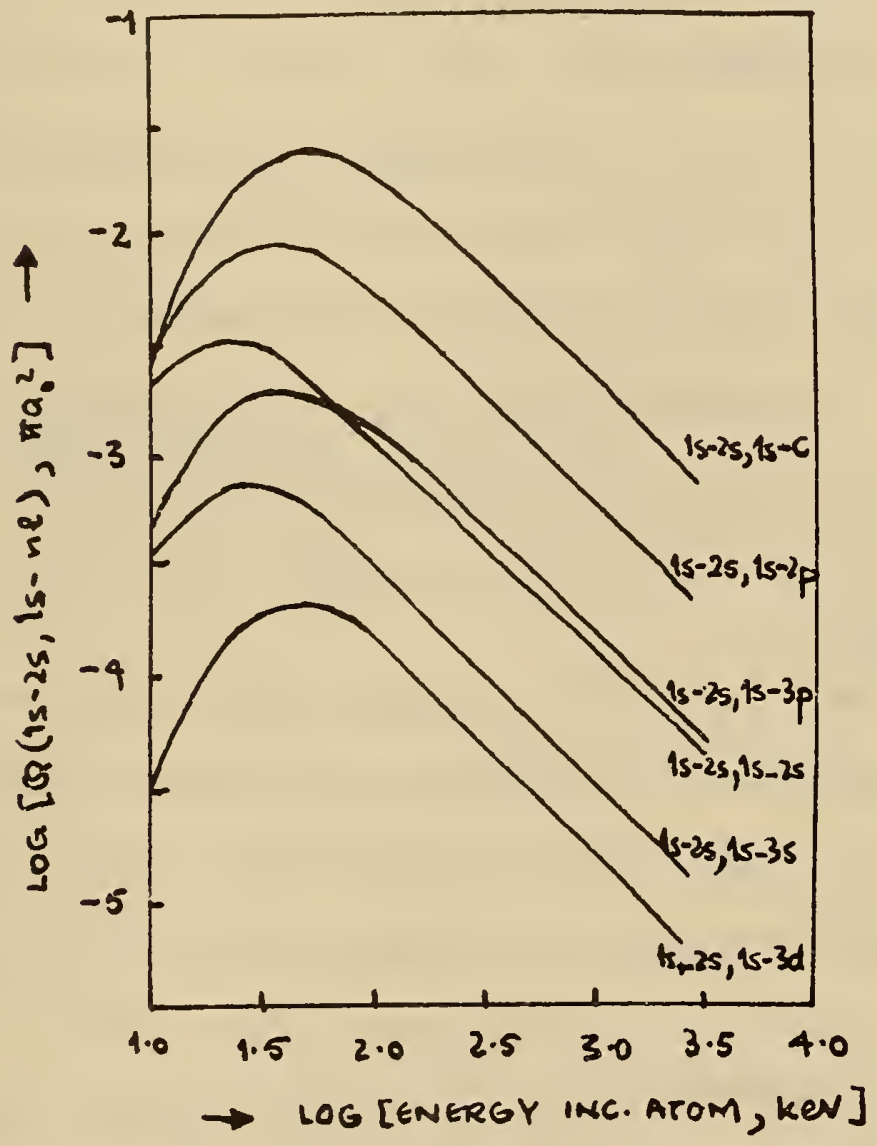


TABLE 2.1

A list of processes for which calculations have been carried out to the lst Born approximation (not including charge transfer).

\section{Proton Excitation}

1) $\mathrm{H}^{+}+\mathrm{H}(1 \mathrm{~s}) \rightarrow \mathrm{H}^{+}+\mathrm{H}(2 \mathrm{~s}, 2 \mathrm{p}, 3 \mathrm{~s}, 3 \mathrm{p}, 3 \mathrm{~d}, \mathrm{C})$.

2) $\mathrm{H}^{+}+\mathrm{He}\left(1 \mathrm{~s}^{2}\right) \rightarrow \mathrm{H}^{+}+\mathrm{He}\left(1 \mathrm{~s} 2 \mathrm{p}\right.$ or $\left.1 \mathrm{~s} 3 \mathrm{p}^{\prime} \mathrm{P}\right)$.

3) $\mathrm{H}^{+}+\mathrm{Na}(3 \mathrm{~s}) \rightarrow \mathrm{H}^{+}+\mathrm{Na}(3 \mathrm{p})$.

\section{Atom - Atom Excitation}

4) $\mathrm{H}(1 \mathrm{~s})+\mathrm{H}(1 \mathrm{~s}) \rightarrow \mathrm{H}(1 \mathrm{~s}, 2 \mathrm{~s}, 2 \mathrm{p}, \mathrm{C})+\mathrm{H}(2 \mathrm{~s}, 2 \mathrm{p}, 3 \mathrm{~s}, 3 \mathrm{p}, 3 \mathrm{~d}, \mathrm{C})$

5) $\mathrm{H}(1 \mathrm{~s})+\mathrm{He}\left(1 \mathrm{~s}^{2}\right) \rightarrow \mathrm{H}\left(\sum\right)+\mathrm{He}\left(1 \mathrm{~s} 2 \mathrm{p}^{\prime} \mathrm{P}\right)$

5!) $\mathrm{He}^{+}(1 \mathrm{~s})+\mathrm{He}\left(1 \mathrm{~s}^{2}\right) \rightarrow \mathrm{He}^{+}(\Sigma)+\mathrm{He}\left(1 \mathrm{~s} 2 \mathrm{p}^{\prime} \mathrm{P}\right)$

6) $\mathrm{H}(1 \mathrm{~s})+\mathrm{He}\left(2 \mathrm{~s}^{3} \mathrm{~s}\right) \rightarrow \mathrm{H}(\Sigma)+\mathrm{He}\left(2 \mathrm{p}\right.$ or $\left.3 \mathrm{p}^{3} \mathrm{P}\right)$ $\mathrm{Ne}(1 \mathrm{~s})+\mathrm{He}\left(2 \mathrm{~s}^{3} \mathrm{~S}\right) \rightarrow \mathrm{Ne}(\Sigma)+\mathrm{He}\left(2 \mathrm{p}\right.$ or $\left.3 \mathrm{p}^{3} \mathrm{P}\right)$

\section{Ionization}

7) $\mathrm{H}^{+}\left(\right.$or $\left.\mathrm{He}^{++}\right)+\mathrm{He} \rightarrow \mathrm{H}^{+}\left(\right.$or $\left.\mathrm{He}^{++}\right)+\mathrm{He}^{+}(1 \mathrm{~s})+\mathrm{e}$

8) $\mathrm{H}^{+}+\mathrm{He}\left(1 \mathrm{~s}^{2}\right) \rightarrow \mathrm{H}^{+}+\mathrm{He}^{+}(2 \mathrm{~s}, 2 \mathrm{p}, 3 \mathrm{p}, 3 \mathrm{~d})+\mathrm{e}$

9) $\mathrm{H}^{+}+\mathrm{Li}\left(1 s^{2} 2 \mathrm{~s}\right) \rightarrow \mathrm{H}^{+}+\mathrm{Li}^{+}\left(1 \mathrm{~s}^{2}\right)+\mathrm{e}$

10) $\mathrm{H}^{+}+\mathrm{Ne} \rightarrow \mathrm{H}^{+}+\mathrm{Ne}^{+}+\mathrm{e}$

IV. Double Ionization

11) $\mathrm{He}^{+}(1 \mathrm{~s})+\mathrm{H}(1 \mathrm{~s}) \rightarrow \mathrm{He}^{++}+\mathrm{H}(\Sigma)+\mathrm{e}$

12) $\mathrm{He}\left(1 s^{2}\right)+\mathrm{H}(1 s) \rightarrow \mathrm{He}\left(\sum\right)+\mathrm{H}^{+}+\mathrm{e}$ 
TABLE 2.1 (Con't.)

\section{Electron Loss}

13) $\mathrm{H}(1 \mathrm{~s})+\mathrm{H}^{-}\left(1 \mathrm{~s}^{2}\right) \rightarrow \mathrm{H}(1 \mathrm{~s}$ or $2 \mathrm{p})+\mathrm{H}(1 \mathrm{~s}$ or $2 \mathrm{p})+\mathrm{e}$

14) $\mathrm{He}\left(1 s^{2}\right)+\mathrm{H}^{-}\left(1 s^{2}\right) \rightarrow \mathrm{He}\left(1 s^{2}\right)+\mathrm{H}(1 s)+\mathrm{e}$

\section{REFERENCES}

1) Bates and Griffing, Proc. Phys. Soc. 2 A, 66, 961 (1953).

2) Moisiewitisch and Stewart, Proc. Phys. Soc. A, 67, 1069, (1954).

3) Bell and Skinner, In Press. (1962).

4) Bates and Griffing, Proc. Phys. Soc. A, 67, 663. (1954)

5) Moisiewitisch and Stewart, (1oc. cit) (1954)

6) Adler and Moisiewitsch (1957) Proc. Phys. Soc. A, 므, 117.

7) Erskine, Proc. Roy. Soc. A, 224, 362. (1954). Mapleton, Phys. Rev., 109, 1166 , (1958).

Grospun and V. d Waale, Nuovo Cimento 19, 872 (1961).

8) Dalgarno and McDowe11, Airglow and Aurorae, p. 341 (1956). Mapleton, (1oc. cit) (1958).

9) McDowell and Peach, Phys. Rev. 121, 1383.

10) Bates, McDowe11 and Omho1t, J.A.T.P. 10, 51, (1957).

11) Boyd et a1., Proc. Phys. Soc. A 70, 110, (1957).

12) Bates and Williams, Proc. Phys. Soc. $\underline{\text { A }}$ 므, 306 (1959).

13) McDowe11 and Peach, Proc. Phys. Soc. 74, 463 (1959).

14) Sida, Proc. Phys. Soc. A 68, 240. (1955). 


\section{LECTURE III}

\section{Slow Charge Transfer}

§1. The Adiabatic Approximation

We suppose that below the maximum predicted by the adiabatic hypothesis that the projectile and the target particle may be considered as forming a quasi-molecule, the velocity of relative motion being such that the electron, considered as a classical particle, would complete several orbits while the nuclear separation changed inappreciably. Then we consider that the quasi-molecule makes transitions (infrequently; $a_{n s} \ll 1$, all s) under the perturbation of the relative motion.

Let the total Hamiltonian be

$$
\mathrm{H}=\mathrm{H}_{0}+\mathrm{V}(\underline{\mathrm{r}}, \underline{\mathrm{R}})
$$

so the system is described by a state function $\Psi_{\mathrm{n}}$ satisfying

$$
\left(H-1 \frac{\partial}{\partial t}\right) \Psi_{n}(\underline{R}, \underline{r})=0
$$

Now at some fixed $R$, the quasi-molecule has eigen states $\chi_{s}(\underline{R}, \underline{r})$ with eigen energies $\epsilon_{S}(R)$ such that

$$
H X_{s}(\underline{R}, \underline{r})=E_{S} X_{s}(\underline{R}, \underline{r}) .
$$

Then expand

$$
\psi_{n}=\int_{s} c_{n s}(t) \chi_{s}(r, R) \exp \left\{-i \int^{t} \varepsilon_{s}(R) d t^{\prime}\right\}
$$


23

and substitute (4) in (2). We obtain

$$
\begin{aligned}
H \int_{s} C_{n s} x_{s} \exp \left\{-i \int^{t} \varepsilon_{s} d t^{\prime}\right\} & =i \int_{s} C_{n s} x_{s} \exp \left\{-i \int^{t} \varepsilon_{s} d t^{\prime}\right\} \\
& +\int_{s} c_{n s} x_{s} \varepsilon_{s} \exp \left\{-i \int^{t} \varepsilon_{s} d t^{\prime}\right\} \\
& +i \int_{s} c_{n s} \frac{\partial x_{s}}{\partial t} \exp \left\{-i \int^{t} \varepsilon_{s} d t^{\prime}\right\}
\end{aligned}
$$

Multiply on the left by

$$
\chi_{m}^{*} \exp \left\{+i \int^{t} \varepsilon_{m} d t^{\prime}\right.
$$

and integrate over the electron coordinates, assuming that

$$
\int x_{s} x_{m}^{*} \exp \left\{i \int^{t} \Delta \varepsilon_{m s} d t^{\prime}\right\} d r=\delta_{m s}
$$

to obtain

$$
\dot{C}_{n m}=-\int_{s} C_{n s}\left\langle m\left|\frac{\partial}{\partial t}\right| s\right\rangle \exp \left\{-i \int^{t} \Delta \varepsilon_{s m} d t^{\prime}\right\}(3.7)
$$

Now

$$
\frac{\partial X_{s}(\underline{R}, \underline{r})}{\partial t}=\frac{\partial X_{s}}{\partial \underline{\underline{R}}} \cdot \frac{\partial \underline{R}}{\partial t}
$$

so (3.7) may be written

$$
\dot{C}_{u m}=-v \cdot \int_{s}^{l} C_{n s}(t)\left\langle m\left|\nabla_{R}\right| \delta\right\rangle \exp \left\{-i \int_{\Delta_{s m(3.9)}^{t}} d t^{\prime}\right\}
$$

which is the P.S.S. approximation in its simplest form. 
[Choosing the (arbitrary) ${ }^{(1)}$ phase of $X_{S}(\underline{r}, \underline{R})$ such that

$$
\frac{x_{s}-x_{s}^{*}}{x_{s}+x_{s}^{*}}=f(\underline{r}) \quad \text { only }
$$

and using the result $\nabla_{\mathrm{R}} \int \mathrm{x}_{\mathrm{s}}{ }^{*} \mathrm{x}_{\mathrm{s}} \mathrm{dr} \equiv \mathrm{o}$, we have

$$
\left\langle s\left|\nabla_{R}\right| S\right\rangle=0
$$

Now suppose that

$$
C_{n s}(-\infty)=\delta_{n s}
$$

as our boundary condition, and assume that we can put $\mathrm{C}_{n s}(t)=$ $C_{n s}(-\infty)$ on the r.h.s. of (3.9). Then we obtain the uncoupled equation, (2)

$$
C_{n m}^{0}=-\underline{v} \cdot\left\langle m\left|\nabla_{R}\right| n\right\rangle \exp \left\{-i j^{t} \Delta \varepsilon_{m n} d t^{\prime}\right\}
$$

which may be integrated to yield

$$
C_{n m}(+\infty)=-\int_{-\infty}^{+\infty} v \cdot\left\langle m\left|\nabla_{R}\right| n\right\rangle \exp \left\{-i \int^{t} \Delta \varepsilon_{m n} d t^{\prime}\right\} d t(3.13)
$$

and

$$
Q_{n m}=2 \pi \int_{0}^{\infty}\left|c_{u m}(+\infty)\right|^{2} p d p
$$

These equations are appropriate only in the absence of coupling, and even the coupled equations (3.9) are inappropriate if cond. (3.6) is not satisfied. In the limit of high velocities we may obtain the impact parameter version of the first Born from this by 
using 1st order perturbed eigenfunctions on (3.13) and the unperturbed eigenenergies.

§ 2. Charge Transfer

We now consider the process

$$
A+(B+e)_{n} \rightarrow(A+e)_{m}+B
$$

in which an electron is transferred from state $n$ of $B$ to state $m$ of A. We deal first with the symmetric resonance case in which

$$
A=B, m=n \text {. }
$$

If the atom (B+e) is in state $\phi_{S}$ as $R \rightarrow \infty$ the corresponding molecular eigenfunctions are $\mathrm{x}_{s}^{+},-$, even and odd with respect to interchange of the nuclei. The corresponding L.C.A.O. approximations to the total state function are

$$
\psi_{1,2}=\frac{1}{\sqrt{2}}\left[x_{s}^{+} \pm x_{s}^{-}\right]
$$

where $\Psi_{1}$ corresponds to the electron on nucleus $B$ as $R \rightarrow \infty$, $\Psi_{2}$ to nucleus A. Adopting the co-ords. shown in Fig. 3.1 
FIG. 3.1

Co-ordinates For The Exchange Problem

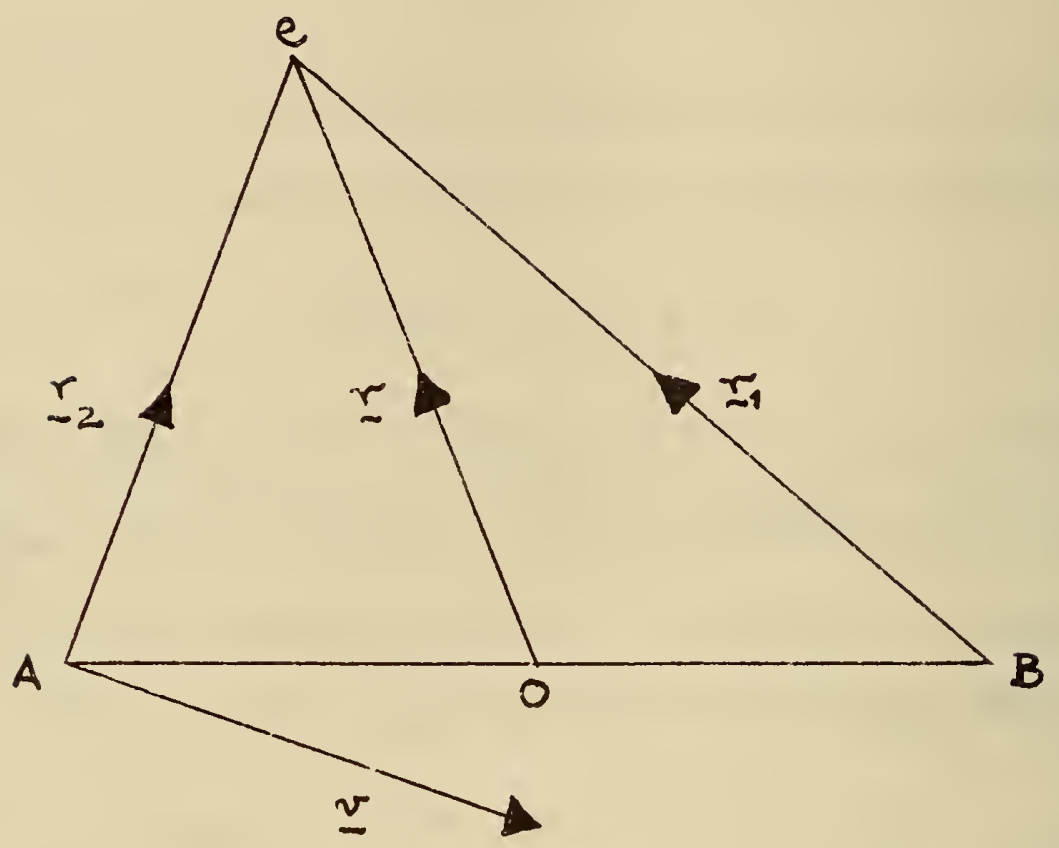


27

and letting $z=v t$ determine the origin of $t$, we have (3)

$$
z-v t=z_{1}-z-\frac{1}{2} z=z_{2}-z+\frac{1}{2} z=0
$$

We now choose an expansion $(1,2)$

$$
\psi=\int_{s} c_{s}^{+} x_{s}^{+}+c_{s}^{-} x_{s}^{-}
$$

where

$$
\begin{aligned}
x_{s}^{ \pm}= & \left\{\left(x_{s}^{+}+x_{s}^{-}\right) e^{-i k z / 2} \pm\left(x_{s}^{+}-x_{s}^{-}\right) e^{+i k z / 2}\right\} . \\
& =\exp \left\{-i \int^{t}\left(\varepsilon_{s}^{ \pm}+\frac{1}{8} m v^{2}\right) d t^{\prime}\right\}
\end{aligned}
$$

Then

$$
\begin{aligned}
\left(H-i \frac{\partial}{\partial t}\right) X_{s}^{ \pm} & =i\left\{\begin{array}{l} 
\pm\left(\varepsilon_{s}^{+}-\varepsilon_{s}^{-}\right) x_{s}^{ \pm} \sin \left(\frac{1}{2} k z\right) \\
-\frac{1}{2} v\left[\frac{\partial}{\partial z_{I_{1}}}\left(x_{s}^{+}+x_{s}^{-}\right) e^{-i k z / 2} \pm \frac{\partial}{\partial z_{I_{2}}}\left(x_{s}^{+}-x_{s}^{-}\right) e^{+i k z / z}\right]
\end{array}\right\} \\
& \cdot \exp \left\{-i \int^{t}\left(\varepsilon_{s}^{ \pm}+\frac{1}{8} m v^{2}\right) d t^{\prime}\right\}
\end{aligned}
$$

such that

$\left(H-i \frac{\partial}{\partial t}\right) X_{S}^{t}=0$ when $\underline{v}=0$ for al $R$, [To prove this note that or when $R \rightarrow \infty$ for all $\Psi$

$$
H X_{s}^{ \pm}=\varepsilon_{s} \pm X_{s}^{ \pm}
$$

and that

$$
H e^{ \pm i k 3 / 2}=\frac{1}{8} m v^{2} e^{ \pm i k z / 2}
$$

]. 
28

We now impose the boundary conditions

$$
c_{n}^{ \pm}(-\infty)=\frac{1}{\sqrt{2}} ; c_{s}^{ \pm}(-\infty)=0, \quad s \neq n
$$

and make the assumption that transitions are infrequent

$$
\underset{s \neq n}{C_{s \neq n}^{ \pm}}(t) \doteq 0
$$

Then from

$$
\left(H-i \frac{\partial}{\partial t}\right) \psi=0
$$

we have

$$
\begin{gathered}
i \sum_{s} \dot{C}_{s}^{+} \int\left(x_{j}^{+}\right)^{*} X_{s}^{+} d r+i \sum_{s} \dot{C}_{s}^{-} \int\left(X_{j}^{+}\right)^{*} X_{s}^{-} d r \\
=c_{j}{ }^{+} T_{j}^{+}
\end{gathered}
$$

where

$$
T_{j}^{+}=\int\left(x_{j}{ }^{*}\right)^{*}\left[H-\underline{i} \frac{\partial}{\partial t}\right] x_{j} d r
$$

clearly

$$
\int\left(x_{j}^{+}\right)^{*}\left(x_{s}^{-}\right) d r=0 \text {, alts }
$$

and if we further assume (incorrectly) [equiv. to assuming

$$
\left.\mathrm{C}_{\mathrm{s}}^{+}=0, \mathrm{~s} \neq \mathrm{j}\right]
$$

$$
\int\left(x_{j}^{+}\right)^{*} x_{s}^{+} d_{r}=\delta_{j s} N_{j s}^{+}
$$

we obtain

$$
i \dot{C}_{j}^{+}=\left(N_{j j}^{+}\right)^{-1} C_{j}^{+} T_{j}^{+}
$$


Now

$$
N_{j j}^{+}=1+\sigma_{j j}^{+}+\frac{1}{2} i \int\left\{x_{i}^{-*} \cdot x_{j}^{+}-x_{j}^{+*} x_{i}^{-}\right\} \sin v z^{d r}(3.2 .15)
$$

The last term of this equation vanishes by symetry arguments to leave the Bates - McCarroll result

$$
\begin{aligned}
& i \dot{C}_{j}^{+}=a_{j}^{+} T_{j}^{+} /\left(1+\sigma_{j}^{+}\right) \\
& \sigma_{j}^{+}=\frac{1}{2} \int\left(X_{j}^{+*} x_{j}^{+}-x_{j}^{-*} x_{j}^{-}\right) \cos v z d r
\end{aligned}
$$

Retaining only the symetric part of $\mathrm{T}_{j}{ }^{+}$one may then integrate this to get the transfer probability in the form.

$$
P=\sin ^{2} \bar{\xi}(p)
$$

where

$$
\xi(p)=\frac{1}{2}\left[\left(\beta^{-}-\beta^{+}\right)+\frac{1}{v} \int_{-\infty}^{+\infty} \Delta \varepsilon_{n}^{+i-} d z\right]
$$

and

$$
\beta^{ \pm}=-\frac{1}{v} \int_{-\infty}^{+\infty}\left\{T_{\text {sym }}^{ \pm} /(1 \pm \sigma)\right\} d z
$$

If $\mathrm{v}$ is small, so is $\left(\beta^{-}-\beta^{+}\right)$, and the result reduces to the P.8.S. method. In general, the effect of the $\left(\beta^{-}-\beta^{+}\right)$term is to reduce the cross section below the P.S.S. value. For the resonant $\mathrm{H}^{+}$on $\mathrm{H}$ case, the effect is noticeable at $2 \mathrm{kev}$, and significant above $25 \mathrm{kev} .(4,5)$ Excellent agreement with the experimental results of Fite et al. ${ }^{(6)}$ is obtained by normalizing 
Fite's result to the theoretical value at the lowest measured energy. Above $15 \mathrm{kev}$ Ferguson's calculations give a result decreasing much more rapidly than the experimental values, but inclusion of coupling to the $2 \mathrm{p}$ state ${ }^{(7)}$ gives a marked improvement (Fig. 3.2). 


\section{REFERENCES}

1. Bates, D. R., (1962), Atomic and Molecular Processes, loc. cit.

2. Bates, D. R., (1961), Quantum Theory, [Ed. Bates] Ch. 8 .

3. Bates, D. R., and McCarroll, R., (1958), Proc. Roy. Soc. A 245 , 175, (1962) Adv. Phys. 11, 1 .

4. Ferguson, A., (1961), Proc. Roy. Soc. A, 246, 540.

5. McCarroll, R., (1961), Proc. Roy. Soc. A, 246, 547.

6. Fite, W. L., et al., (1960) Phys. Rev. 119, 663.

7. McElroy, M. B., (1962), To be published. 


\section{CAPTION TO FIGURE 3.2}

The solid curve shows Ferguson's calculated values of $Q^{\text {trans }}$ for $\mathrm{H}^{+}$in $\mathrm{H}$, the dashed curve the effect of including coupling to $2 \mathrm{p}$. (McElroy 1962). The $x ' s$ are the experimental points renormalized (o's) to the P.S.S. calculation at $1 \mathrm{kev}$. 
FIG. 3.2

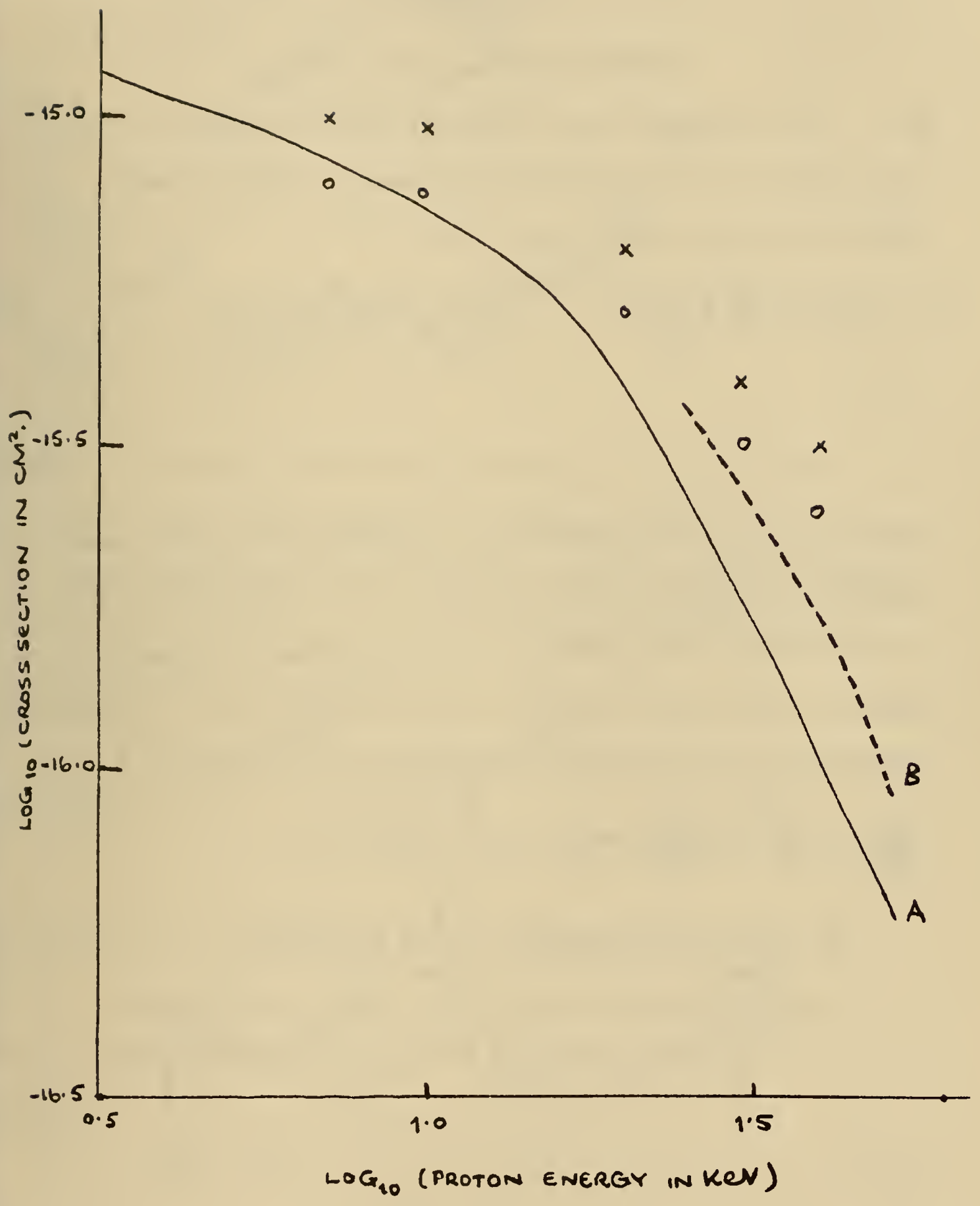




\section{LECTURE IV}

\section{Charge Transfer In Other Cases}

§1. The treatment given in Lecture III can readily be generalized to take account of non-symmetric charge transfer. Expanding the total wave function as

$\Psi_{n}=\sum_{p} a_{p}(t) \phi_{p}^{A}\left(\frac{r}{\underline{R}}\right) e^{-i \int^{t} \eta_{p} d t^{\prime}}+\sum_{q} b_{q}(t) \phi_{q}^{B}\left(\frac{r}{R}\right) e^{-i \int \eta_{q}^{t} d t^{\prime}}$

where $\phi_{p}^{A}, \phi_{q}^{B}$ are proportional to eigenfunctions of the quasimolecule $A B$ with the active electron on $A$ or $B$ respectively when $R \rightarrow \infty$. Noting that $\phi_{p}^{A}$ and $\phi_{q}^{B}$ are not in general orthogonal (in the absence of exact wave functions) one can derive a set of differential equations for the occupation coefficients of the form $(1)$

$$
\begin{aligned}
\frac{\partial a_{i}}{\partial z}+ & \sum_{q} s_{i q} \frac{\partial b_{q}}{\partial z} \exp \left\{-\frac{i}{v} \int_{-\infty}^{z} \Delta \eta_{i q} d z^{\prime}\right\} \\
= & -\sum_{p \neq i} a_{p} L_{i p} \exp \left\{-\frac{i}{v} \int_{-\infty}^{z} \Delta \eta_{p i} d^{\prime} \cdot\right\} \\
& -\sum_{q} b_{q} M_{i q} \exp \left\{\frac{i}{2} v z-\frac{i}{v} \int_{-\infty} \Delta \eta_{q i} d z^{\prime}\right\}
\end{aligned}
$$


35

together with similar equations for the $b_{j}$, where

$$
\begin{aligned}
& s_{i q}=\int X_{i}^{A *} X_{q}^{B} e^{i v z} d r \\
& L_{i p}=\int X_{i}^{A *} \nabla_{r_{2}} X_{p}^{A} d r \\
& M_{i q}=\int X_{i}^{A * \nabla_{r_{2}}} X_{p}^{B} e^{i v z_{2}} d r
\end{aligned}
$$

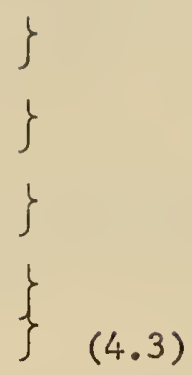

and

$$
\phi_{p}^{A}=x_{p}^{A} e^{-\frac{i}{2} v z} e^{-\frac{i}{8} v^{2} t}
$$

Clearly the coupling terms involving the $\mathrm{M}_{i q}$ are of considerable importance. This set of equations has so far proved intractable.

A two state approximation which should be valid at somewhat higher energies has been developed by several authors, $(1,2,3,4)$ and will now be described. We expand $(1)$

$$
\Psi_{n}=a_{i} \psi_{i}^{A} \cdot(r) e^{-i \varepsilon_{i}^{A} t}+b_{j} \psi_{j}^{B} e^{-i \varepsilon_{j}^{B} t}+\gamma(r, t)
$$

where $\gamma(\underline{r}, t)$ is orthogonal to both the $a_{i}$ and $b_{j}$ term and the $\psi_{i}^{A}, \psi_{i}^{B}$ are proportional to the initial and final unperturbed atomic eigenfunctions. Now

$$
\left(H-i \frac{\partial}{\partial t}\right) \Psi_{n}=0
$$


36

so substituting (4.5) we get a set of coupled equations

$$
\begin{aligned}
& i\left\{\dot{a}_{i}+\dot{b}_{j} s_{i j} e^{-i \Delta \varepsilon_{j j} t}\right\}=b_{j} k_{i j} e^{-i \Delta \varepsilon_{j i} t}+\sum_{p} a_{p} H_{i j} e^{-i \Delta \varepsilon_{j i} t} \\
& i\left\{b_{j}+a_{i} s_{j i} e^{-i \Delta \varepsilon_{j} t}\right\}=a_{i} k_{j i} e^{-i \Delta \varepsilon_{i j} t}+\sum_{q} b_{q} H_{j q} e^{-i \Delta \varepsilon_{j} g^{t}}
\end{aligned}
$$

with

$$
\left.\begin{array}{ll}
s_{i j}=\int \phi_{i}^{A *}\left(\underline{r}_{1}\right) \phi_{j}^{B}\left(\underline{r}_{2}\right) e^{i v z} d r &
\end{array}\right\}
$$

and $V^{A}, V^{B}$ are the initial and final unperturbed potentials.

We have taken the atomic eigenfunction to be

$$
\begin{gathered}
\phi_{i}^{A} \text { and } \phi_{j}^{B} \text {, where } \\
\psi_{1}^{A}=\phi_{1}^{A} \exp \left\{-\frac{i}{2} v z-\frac{i}{8} v^{2} t\right\} \\
\left.\psi_{j}^{B}=\phi_{j}^{B} \exp \left\{\frac{\dot{v}}{2} v z-\frac{i}{3} v^{2} t\right\}\right\}
\end{gathered}
$$

Making the usual two state approximation

$$
\lambda_{i}(-\infty)=1 ; \lambda_{j}(-\infty)=b_{j}(-\infty)=0 \quad(j \neq i)
$$

$$
\begin{aligned}
& \text { and assuming transitions are rare, gives }-i \Delta \varepsilon_{j i} t \\
& \left.i\left\{a_{i}+b_{j} S_{i j} e^{-i \Delta \varepsilon_{j i} t}\right\}=b_{j} k_{i j} e^{-i \Delta \varepsilon_{i j} t}\right\}=a_{i} k_{j i} e^{-i \Delta \varepsilon_{i j} t}+b_{j} H_{j j}
\end{aligned}
$$


37

Multiplying the lower equation by $s_{i j} e^{-i \Delta \epsilon_{j i} t}$ on the right, and subtracting

$$
\begin{aligned}
& i\left\{1-\left|s_{i j}\right|^{2}\right\} a_{v}=a_{i}\left(H_{i i}-s_{i j} k_{j i}\right)+b_{j}\left(k_{j}-s_{j j} H_{j j}\right) e_{(4.12 a)}^{-i \Delta \varepsilon_{j i} t} \\
& \text { and similarly } \\
& i\left\{1-\left|s_{i j}\right|^{2}\right\} b_{j}^{\prime}=b_{j}\left(H_{j j}-s_{j i} k_{i j}\right)+a_{i}\left(k_{j i}-s_{j i} H_{i i}\right) e_{(4.12 b)}^{-i \Delta \varepsilon_{i j} t}
\end{aligned}
$$

If we note that the imaginary parts of

$$
x_{i}=\frac{H_{i}-s_{i j} k_{j i}}{1-\left|s_{i j}\right|^{2}}, \beta_{j}=\frac{H_{j j}-S_{j i} k_{i j}}{1-\left|s_{i j}\right|^{2}}
$$

are antisymmetric in $t$, we may remove the secular terms by putting

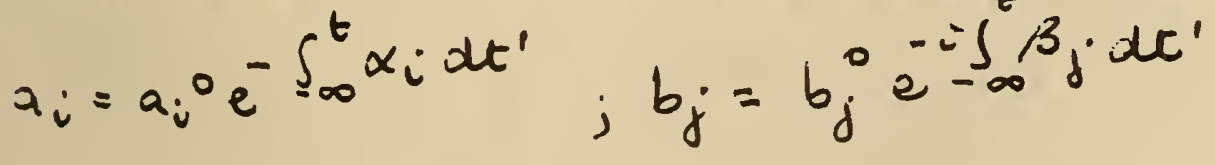

to obtain

$$
\begin{aligned}
& i \dot{x}_{i}^{0}=b_{j}^{0}\left[\frac{k_{i j}-s_{i j} H_{i j}}{1-\left|S_{i j}\right|^{2}}\right] \exp \left[-i \Delta \varepsilon_{j i} t+i \dot{\delta}_{i j}\right] \\
& i \dot{b}_{j}^{0}=\lambda_{i}^{0}\left[\frac{k_{j i}-S_{j i} H_{i i}}{1-\left|S_{i j}\right|^{2}}\right] \exp \left[-i \Delta \varepsilon_{i j} t-i \delta_{i j}\right]
\end{aligned}
$$

where

$$
\delta_{i j} \doteqdot \int_{-\infty}^{t}\left(H_{i i}-H_{j j}\right) d t^{\prime}
$$


and makes allowance for distortion. In the symmetric resonance case $\delta_{i j} \equiv o$, and the equations uncouple to give

$$
Q=2 \pi \int_{0}^{\infty}\left|b_{j}(\infty)\right|^{2} p d p
$$

where

$$
\left|b_{j}(\infty)\right|^{2}=\sin ^{2}\left\{\int_{-\infty}^{+\infty} M_{i j} d t\right\}
$$

and

$$
M_{i j}=\left[\frac{k_{j i}-5 j i H_{i}}{1-\left|S_{i j}\right|^{2}}\right] \exp \left\{-i \Delta \varepsilon_{i j} t\right\}
$$

However, we must generally make the simplifying assumption $b_{j}(t)<a_{i}(t)(a 11 t)$, and we then obtain

$$
\left|b_{j}(\infty)\right|^{2}=\left|\int_{-\infty}^{t \infty} M l_{i j}^{1} d t\right|^{2}
$$

with

$$
M_{i j}^{\prime}=M_{i j} e^{-i \delta_{i j}}
$$

These equations have been applied to

$$
H_{e}^{+t}+H(15) \rightarrow H_{e}^{+}(15)+H^{+}
$$

by McCarrol1 and McElroy ${ }^{(6)}$ and to

$$
\mathrm{H}^{++}+\mathrm{H}(\mathrm{s}) \rightarrow \mathrm{H}_{e}^{+}\left(2 \mathrm{~s}, 2 p_{x}, 2 p_{z}\right)+H+
$$

by McElroy (7) and the results are tabulated below. The agreement with the results of Fite et a1.(8) and the data taken in molecular hydrogen $(9)$ is shown in Fig. 4.1. It is far from clear whether 
Bates and Lynn's ${ }^{(10)}$ prediction of a rapid decrease in the cross section for the accidental resonance case as $\mathrm{v} \rightarrow \mathrm{O}$ is confirmed. Probably coupling is as least as important an effect as distortion. Equations (4.15) reduce to those of Bassel and Gerjnoy(11) if the $\left|S_{i j}\right|^{2}$ term is omitted.

\$2. Comparison of the Various Treatments

If in the result (3.38) we ignor the $\beta \pm$ term, then the P.S.S. approximation yields for the cross section for symmetric resonance charge transfer

$$
Q=2 \pi \int_{0}^{\infty} p \sin ^{2} \xi(p) d p
$$

with

$$
\xi=\xi_{0}=\frac{1}{v} \int_{\phi}^{\infty} \frac{\Delta \varepsilon^{t,}(R) R d R}{\left(R^{2}-\phi^{2}\right)^{1 / 2}}
$$

$$
\xi=\bar{\xi}_{1}=\int_{-\infty}^{+\infty} \frac{\left(k_{i j}-s_{i j} H_{j j}\right)}{\left(1-\left|s_{i j}\right|^{2}\right)} d t
$$

If we ignore the $e^{-i v z}$ term $(v \rightarrow 0)$ and take $\left|s_{i j}\right|^{2}<<1$, we have

$$
\xi_{1} \div \frac{1}{v} \int_{\rho}^{\infty} \frac{\left(k_{i j}-H_{j j}\right)}{\left(R^{2}-p^{2}\right)^{1 / 2}} R d R
$$

and

$$
k_{i j}-H_{j j} \doteq\left\langle A\left|v^{A}\right| B\right\rangle-\left\langle A\left|v^{B}\right| A\right\rangle
$$

which differs from (2) only in that the molecular eigenenergies 
are replaced by their L.C.A.O. approximations. Result ${ }^{(3)}$ in the limit $e^{-i v z} \rightarrow 1$ has been obtained in a simple fashion by Gurnee and Magee (2) and their method has recently been extended to the asymmetric case by Rapp and Francis ${ }^{(4)}$, who ignor the exponential in (4.9). They show that in this approximation asymmetric resonance charge transfer should show the typical

$$
\sqrt{Q}=A \log E+B
$$

behavior down to some minimum energy $E_{0}$ which depends on the energy to defect $\Delta \mathrm{E}_{\text {if }}$ in a complicated fashion. For $\mathrm{E}<\mathrm{E}_{O}$, the usual adiabatic variation of the cross section is obtained. 


\section{REFERENCES}

1. Bates, D. R. and McCarrol1, R., (1962), Adv. Phys. 11, 1 .

2. Gurnee and Magee, (1957), J. Chem. Phys. 26, 1237.

3. Mittleman, M., (1961), Phys. Rev. 122, 499.

4. Rapp and Francis, (1962), J. Chem. Phys. (In Press).

5. McCarrol1, R., (1961), Proc. Roy. Soc. A. 246, 547.

6. McCarrol1, R. and McElroy, M. B., (1962), Proc. Roy. Soc. A. (In Press).

7. McElroy, M. B., (1962). To be published.

8. Fite, W., et al., (1960), Phys. Rev. 119, 663.

9. Allison, S. K., (1958), Phys. Rev. 110, 670.

10. Bassel, R. and Gerjnoy, E., (1960), Phys. Rev. 117, 749. 


\begin{tabular}{|c|c|c|c|c|c|c|c|c|c|c|c|}
\hline $\begin{array}{l}I \\
\underline{z} \\
\tilde{u}\end{array}$ & $\begin{array}{l}3 \\
3 \\
0 \\
0 \\
0\end{array}$ & $\begin{array}{l}i \\
\frac{0}{\infty} \\
+ \\
m \\
\dot{m} \\
\dot{d}\end{array}$ & $\mid \begin{array}{c}1 \\
0 \\
x \\
+7 \\
+\end{array}$ & $\begin{array}{l}\frac{\infty}{10} \\
\frac{0}{x} \\
n \\
\underline{z}\end{array}$ & $\begin{array}{c}\frac{\sigma}{i} \\
\frac{0}{x} \\
\nu \\
o \\
\dot{\gamma}\end{array}$ & $\begin{array}{l}\frac{\sigma}{\alpha} \\
\frac{0}{x} \\
\frac{b}{\dot{\alpha}}\end{array}$ & 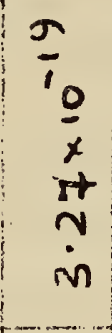 & $\begin{array}{l}\frac{0}{10} \\
\stackrel{0}{x} \\
\frac{x}{2} \\
\stackrel{2}{2}\end{array}$ & $\begin{array}{l}10 \\
\frac{0}{x} \\
b \\
+7 \\
i n\end{array}$ & $\mid \begin{array}{c}0 \\
0 \\
0 \\
x \\
0 \\
\infty \\
\infty\end{array}$ & $\begin{array}{l}10 \\
x \\
m \\
0\end{array}$ \\
\hline 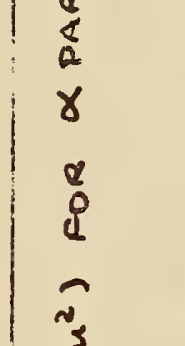 & $\begin{array}{l}3 \\
b \\
b \\
+\end{array}$ & 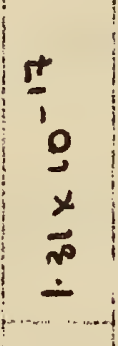 & $\left|\begin{array}{c}0 \\
6 \\
0 \\
2 \\
+ \\
6 \\
6\end{array}\right|$ & \begin{tabular}{l|}
$\infty$ \\
$i$ \\
$\frac{0}{x}$ \\
$N$ \\
$n$ \\
$\dot{n}$
\end{tabular} & $\begin{array}{l}\frac{\sigma}{1} \\
\frac{2}{x} \\
\rho_{n} \\
\omega\end{array}$ & $\begin{array}{l}\frac{m}{1} \frac{0}{x} \\
\frac{\infty}{a} \\
\dot{0}\end{array}$ & 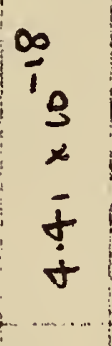 & $\begin{array}{c}\frac{5}{1} \\
\frac{0}{x} \\
\sigma \\
\Phi \\
\end{array}$ & $\begin{array}{c}0 \\
0 \\
0 \\
x \\
0 \\
+ \\
n\end{array}$ & $\begin{array}{c}\frac{n}{0} \\
\frac{0}{x} \\
\frac{0}{4} \\
\dot{0}\end{array}$ & Q \\
\hline & $\begin{array}{l}3 \\
3 \\
b_{3} \\
3\end{array}$ & 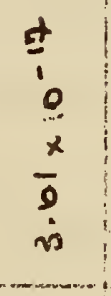 & $\begin{array}{l}5 \\
0 \\
x \\
0 \\
-\end{array}$ & 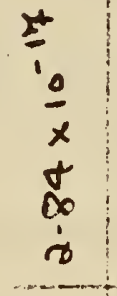 & $\begin{array}{l}\frac{\infty}{0} \\
x \\
\pm \\
0\end{array}$ & $\begin{array}{l}\frac{5}{0} \\
\frac{0}{x} \\
n \\
\dot{d} \\
\dot{d}\end{array}$ & $\begin{array}{l}\frac{1}{1} \\
\frac{0}{x} \\
\frac{0}{i n} \\
n\end{array}$ & $\begin{array}{l}\frac{5}{i} \\
0 \\
x \\
a \\
⿱ 亠 䒑\end{array}$ & $\begin{array}{l}\frac{\pi}{4} \\
\frac{0}{x} \\
2+ \\
= \\
=\end{array}$ & $\begin{array}{c}0 \\
1 \\
x \\
x \\
0 \\
0 \\
+\end{array}$ & 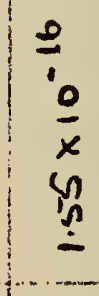 \\
\hline & $\begin{array}{l}3 \\
8 \\
8 \\
\end{array}$ & 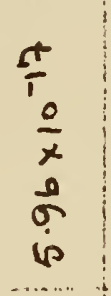 & $\begin{array}{l}\frac{T}{\circ} \\
x \\
f \\
-\end{array}$ & 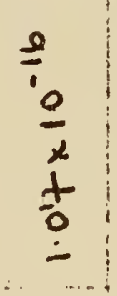 & $\mid \begin{array}{l}\frac{5}{0} \\
\frac{0}{x} \\
0 \\
5 \\
5\end{array}$ & $\begin{array}{l}\frac{0}{10} \\
\frac{\partial}{x} \\
\stackrel{M}{=}\end{array}$ & $\begin{array}{l}2 \\
10 \\
\bar{x} \\
6 \\
m \\
\stackrel{0}{-}\end{array}$ & $\begin{array}{c}0 \\
0 \\
0 \\
x \\
0 \\
0 \\
n\end{array}$ & 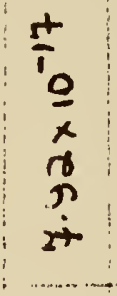 & $\begin{array}{c}\frac{1}{10} \\
\frac{0}{x} \\
\dot{f} \\
\stackrel{0}{-}\end{array}$ & $\overline{\bar{x}}$ \\
\hline & $\begin{array}{l}3 \\
3 \\
0 \\
n\end{array}$ & 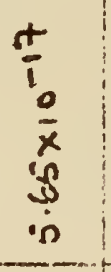 & $\begin{array}{l}\tilde{\omega} \\
\dot{0} \\
\bar{x} \\
0 \hat{N} \\
-\end{array}$ & $\begin{array}{l}\frac{0}{1} \\
\frac{1}{x} \\
6 \\
b \\
b \\
\vdots\end{array}$ & $\begin{array}{l}\frac{0}{1} \\
\frac{0}{x} \\
j \\
+ \\
+\end{array}$ & $\begin{array}{l}\frac{0}{1} \\
\frac{0}{x} \\
\frac{0}{j}\end{array}$ & 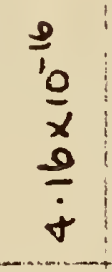 & $\begin{array}{l}\frac{0}{1} \\
\frac{0}{x} \\
0 \\
\vdots \\
n\end{array}$ & $\begin{array}{l}\frac{0}{1} \\
\frac{0}{x} \\
o \\
\stackrel{x}{x}\end{array}$ & \begin{tabular}{l}
$\frac{n}{0}$ \\
\hdashline \\
0 \\
0 \\
$\dot{x}$
\end{tabular} & $\begin{array}{l}\bar{x} \\
+ \\
0 \\
\dot{x}\end{array}$ \\
\hline & $\begin{array}{l}3 \\
y \\
1 \\
\gamma \\
\gamma\end{array}$ & $\begin{array}{l}\frac{\pi}{10} \\
\frac{x}{x 0} \\
\frac{x}{20}\end{array}$ & $\begin{array}{l}\frac{ \pm}{1} \\
0 \\
\frac{0}{x} \\
0 \\
0\end{array}$ & $\begin{array}{l}\frac{2}{10} \\
\frac{0}{x} \\
0 \\
0\end{array}$ & $\begin{array}{l}\frac{0}{1} \\
\frac{0}{x} \\
0 \\
0 \\
\dot{0} \\
\dot{-}\end{array}$ & $\begin{array}{l}\frac{0}{10} \\
\frac{0}{x} \\
\frac{p}{p} \\
\dot{n}\end{array}$ & 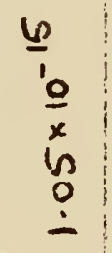 & $\begin{array}{c}\frac{1}{1} \\
\frac{0}{x} \\
+ \\
0 \\
0\end{array}$ & $\begin{array}{l}\frac{0}{1} \\
\frac{0}{x} \\
\hat{w} \\
\dot{p}\end{array}$ & $\begin{array}{l}\frac{6}{10} \\
x \\
0 \\
d \\
d\end{array}$ & $\bar{x}$ \\
\hline & 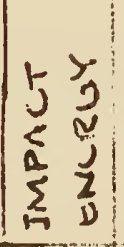 & $\begin{array}{l}\stackrel{\Perp}{=} \\
\mathscr{g}^{\infty}\end{array}$ & $\frac{\tilde{n}}{x}$ & $\frac{x}{\partial}$ & $\frac{\sqrt{\hat{\gamma}}}{\hat{\sigma}}$ & $\underbrace{\frac{x^{x}}{\delta}}_{\dot{\theta}}$ & $\begin{array}{c}\hat{x} \\
\hat{\gamma} \\
\bar{x}\end{array}$ & $\frac{\partial 0}{\partial}$ & $\frac{\widehat{x}}{\frac{\delta}{\partial}}$ & $\frac{\frac{\sigma}{5}}{\tilde{\sigma}}$ & ₹े \\
\hline
\end{tabular}




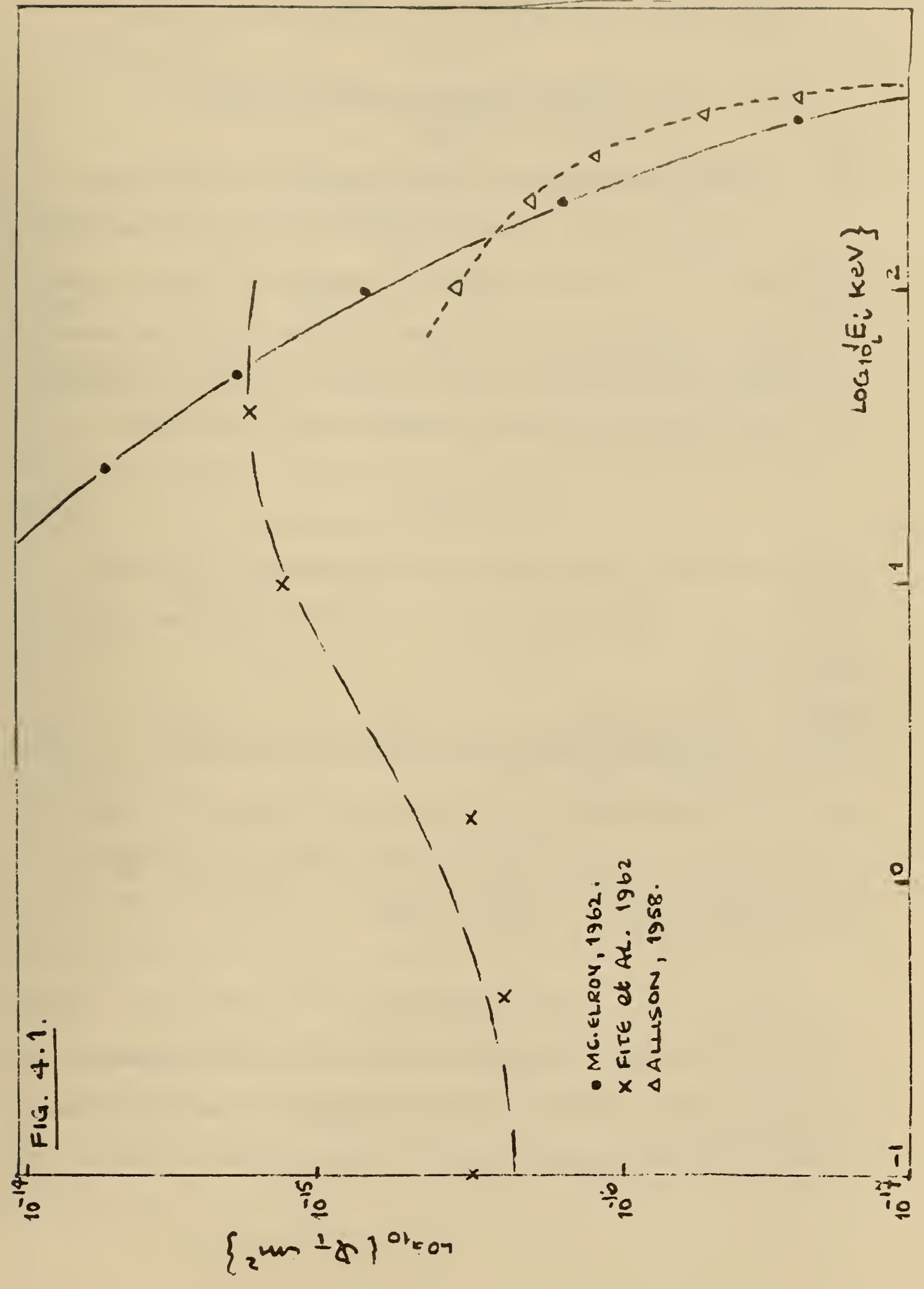


LECTURE V

\section{(i) Further Remarks on Charge Transfer (ii) Curve - Crossing}

S1. Elementary Treatments of Some Validity At High Energies The early work on charge transfer $\left(\mathrm{H}^{+}\right.$on $\left.\mathrm{H}\right)$ by Brinkmann and Kramers $(1,2)$ and by Oppenheimer ${ }^{(3)}$ treated the process in the first Born approximation and retained only the interaction between the incident nucleus and the active electron (or the corresponding past interaction) in the matrix element. That is they took

$$
M=M_{B K}=K_{j i}
$$

which equivalent to the refined fast approximation (4.15) with $S_{i j}=\delta_{i j}=0$. The cross section, to the first order in $(m / M)$ is then given by

$Q_{1 s, 15}^{08 k}=\frac{2^{18} z_{1}^{5} z_{2}^{5} s_{i}^{8}}{3}\left\{s_{i}^{2}+\left(z_{1}+z_{2}\right)^{2}\right\}^{-5}\left\{s i^{2}+\left(z_{1}-z_{2}\right)^{2}\right\}^{-5}$

where $S_{i}^{2}$ is the usual energy parameter and $z_{1}$ and $z_{3}$ are the nuclear charges. Similar expressions may be derived for capture into excited states ${ }^{(4)}$. However, when

$$
Q_{c}=\sum_{n l} Q_{1 s, n l}
$$

for $\mathrm{H}^{+}$on $\mathrm{H}$ is compared with the experimental data ${ }^{(5)}$ taken on $\mathrm{H}_{2}$, (per $\mathrm{H}$ atom) it is found to overestimate by a factor of five at energies up to $400 \mathrm{kev}$. 
Bates and Dalgarno $(4,6)$ and independently Jackson and Schiff ${ }^{(7)}$ suggested that

$$
\mathrm{M}_{\text {BDJS }}=\mathrm{K}_{i j}+\mathrm{V}_{\mathrm{nn}}
$$

where $\mathrm{V}_{\mathrm{nn}}$ is the nuclear interaction

$$
v_{\text {nn }}=-\int \phi_{i}^{A *} \frac{z_{1} z_{2}}{R} \phi_{j}{ }^{B} \text { in }
$$

was a better approximation in practice. However, ${ }^{(8)} \mathrm{v}_{\mathrm{nn}}$ vanishes in the limit $M \rightarrow \infty$. This approximation is equivalent to (4.15) ignoring distortion, neglecting $\left|s_{i j}\right|^{2}$ compared with unity and replacing the resulting Matrix element

$$
\begin{aligned}
M_{B G} & =H_{i i}-k_{i j} \\
& =k_{i j}+v_{n n}-<k_{i j}+v_{n n}>
\end{aligned}
$$

where $<>$ indicates the initial static potential, by (5.4). The resulting $Q_{c}$ for $\mathrm{H}^{+}$on $H$, and on $\mathrm{He}$ are in excellent agreement with experiment over a wide energy range(10).

McDowell et al. are presently extending these calculations to $\mathrm{H}^{+}$on $\mathrm{H}^{-}$and $\mathrm{H}^{+}$on $\mathrm{L}_{i}^{+}$. In these cases Coulomb forces play some role and McDowell ${ }^{(11)}$ has recently shown that the correct matrix element (to the 1st order) in that case is (5.1) where the initial unperturbed eigenfunctions are now taken to be products $\mid U_{i}>$ of atomic orbitals and an incoming Coulomb wave. We have 
incoming Coulomb wave. We have

$$
M^{C B}=\left\langle\psi_{f}\left|V^{A}\left(r_{1}\right)\right| u_{i}\right\rangle
$$

where $\mid U_{t}>$ satisfies

$\left[H_{0}+v^{A}\left(\underline{r}_{1}\right)+v_{n n}\right] u_{i}>=E_{i} \mid u_{i}>$

Now the atomic functions used in Lecture (4) $\mid \phi_{i}{ }^{A}>$ satisfy

$$
\left[H_{0}+V^{A}\left(r_{1}\right)\right] \phi_{i}^{A}>=E_{i}^{\prime}\left|\phi_{i}^{A}\right\rangle
$$

so to the 1 st order

$$
\left.\left.u_{i}\right\rangle=\phi_{i}^{A}\right\rangle+\int_{j} \frac{\left\langle\phi_{i}^{A}\left|v_{u n}\right| \phi_{j}^{A}\right\rangle}{E_{i}-E_{j}}\left|\phi_{j}^{A}\right\rangle
$$

and substituting this in (5.7) we may obtain the correction due to the Coulomb potential as

$$
M^{i B}=M_{B K}+\int_{k} \frac{\left\langle\phi_{i}^{A}\left|v_{n n}\right| \phi_{k}^{A}\right\rangle\left\langle\phi_{k}^{A}\left|V^{A}\left(r_{1}\right)\right| \phi_{j}^{B}\right\rangle}{E_{i}-E_{k}}
$$

That is $V_{n n}$ scatters the incident system from state $i$ into state $k$, and capture proceeds from there to the final state. The important virtual transitions are (i) the target is excited with $\Delta l= \pm 1$, and (ii) the target is unexcited but the relative momentum vector is altered. Both these contributions can be evaluated by the Femann technique, and calculations are in progress. The total /yn effect is sma11, for $\left|M^{c B}\right|^{2}$ can be evaluated in closed form, and 
it may be shown that if (11)

$$
\frac{z_{1}}{k_{i}} \ll 1
$$

then

$$
\left|\mathrm{M}^{\mathrm{CB}}\right|^{2} \div\left|\mathrm{M}_{\mathrm{BK}}\right|^{2}
$$

Returning to (5.6) we may attempt to improve it by including distortion, by noting that the most important effect is the distortion of the unperturbed initial atomic eigenfunction by the incoming particle, i.e. by $\mathrm{V}^{\mathrm{B}}\left(\underline{\underline{r}}_{2}\right)$. McDowe11 ${ }^{(8)}$ has therefore computed the cross section, replacing $\mathrm{M}_{\mathrm{BDJS}}$ by

$$
M_{\text {dist }}=\left\langle\psi_{f}^{B}\left|V_{\text {un }}+v^{B}\left(\underline{r}_{2}\right)\right| X_{i}^{A}\right\rangle
$$

where

$$
\left|x_{i} A\right\rangle=\left|\phi_{i}^{A}\right\rangle+\int_{j}^{\prime} \frac{\left\langle\phi_{i} A\left|V^{B}\left(v_{2}\right)\right| \phi_{j}^{A}\right\rangle}{E_{i}^{\prime}-E_{j}^{\prime}}\left|\phi_{j}{ }^{A}\right\rangle_{(5.15)}
$$

which is equivalent to replacing $\left\langle\mathrm{K}_{i j}+\mathrm{V}_{\mathrm{nn}}>\right.$ in $\left(5.6^{-}\right)$by the sum in (5.15). This modification greatly decreases the cross section at low energies ( $<200 \mathrm{kev}$.$) . Calculations for \mathrm{He}^{++}$on $\mathrm{H}$ by this approximation are now in progress. 


\section{Curve Crossing}

If the Schrodlinger equation of the quasi-molecule formed by the colliding systems is non-separable in the electron coordinates, and the potential energy curves of states $\phi_{1}$ and $\phi_{2}$ are known, then it is possible that $V_{1}(R)$ may equal $V_{2}(R)$ for some $R=R_{x}$ say. If we then proceed to a higher approximation

$$
\Psi_{1,2}=c_{1}^{1,2} \phi_{1}+\bar{c}_{2}^{1,2} \phi_{2}
$$

Then it is found (Moisiewitsch, B. L. 1962) that the new potential energies $V_{1}^{\prime}(R), V_{2}^{\prime}(R)$ are never equal unless $\phi_{1}$ and $\phi_{2}$ belong to different symmetry classes.

Landan $(12)$ and Zener(13) suggested that in such cases transitions between the states corresponding to $\phi_{1}(R-\infty)$ and $\phi_{2}(R \rightarrow \infty)$ were likely only for $R \div R_{x}$. If $P$ is the probability of such a transfer, on any one passage through $R_{X}$, then the transition probability is

$$
\tilde{v}=2 P(1-P)
$$

and is sma11, both when $P$ is small and when $P$ is large. Bates (14) has recently criticized these assumptions, and their method of approximating $\mathrm{P}$, as follows:-

Consider the transition

$$
(A+e)_{n}+B \rightarrow A+(B+e)_{m}
$$

at some impact parameter $\mathrm{p}$, under a perturbation $\mathrm{H}^{\prime}$. 
49

Let

$$
H_{i j}=j \phi_{i}^{*} H^{\prime} \phi_{j} \underline{d r}
$$

be a zero order matrix element, where we take $\phi_{n}$ and $\phi_{m}$ to be appropriate linear combinations of the (exact) molecular eigenfunctions and of the same symmetry class. If $y^{A}$ describes the state in which the electron is on nucleus $A$ as $R \rightarrow \infty$, in the zero th order approximation, then $\Phi_{n}$ is the wave function describing $\left\{(A+e)_{n}+B\right\}$ for $R \geq R_{x}$, but $\left\{A+(B+e)_{m}\right\}$ for $R<R_{x}$, and similarty for $\Phi_{m}$. Thus

$$
\mathrm{H}_{\mathrm{nm}}(\mathrm{R}) \neq 0(\mathrm{a} 11 \mathrm{R}) \text {. }
$$

Further if $\mathrm{H}$ is the total Hamiltonian, (since in general $\mathrm{A}_{\mathrm{n}}=$ $\sum_{j} A_{n j} \phi_{j}$ and in our case the sum runs only over $\left.j=n, m_{*}\right)$,

$$
\begin{aligned}
& H \phi_{n}(\underline{R}, r)=H_{n n}(R) \phi_{n}+H_{m n}(R) \phi_{m} \\
& H \phi_{m}(\underline{R}, r)=H_{n m}(K) \phi_{n}+H_{m m}(R) \phi_{m} \\
& \text { and at } R=R_{x}
\end{aligned}
$$

$$
H_{n n}\left(R_{x}\right)=H_{m n}\left(R_{x}\right)
$$

while

$$
\Delta E_{m n}\left(R_{x}\right)=2\left|H_{n m}\left(R_{x}\right)\right|
$$

We now proceed as in (4.5), taking a two state expansion

[Assumption 0]

$$
\Psi_{n}=c_{n}(t) \Psi_{n}+c_{m}(t) \psi_{m}
$$

with

$$
\psi_{n}=\phi_{n} \exp \left\{-\frac{i}{2} k z-i \int^{t}\left[H_{n u}+\frac{1}{8} m v^{2}\right] d t^{\prime}\right\}
$$


50

and substitute this into

$$
\left\{H-\frac{i}{\partial t}\right\} \psi_{n}=0
$$

A typical term yields

$$
\begin{aligned}
& \left.\begin{array}{rl}
\left\{H-i \frac{\partial}{\partial c}\right\} \psi_{n}= & \left.\left[H_{n u} \phi_{n}+H_{m_{n}} \phi_{n}\right\}\right] \\
& +\left\{H_{n u} \phi_{n}-i v \frac{\partial \phi_{n}}{\partial z} r_{1}\right\}
\end{array}\right] \times \\
& x \exp \left\{-\frac{i}{2} k z-i \int^{t}\left[H_{u n}+\frac{1}{8} m v^{2} \int d t^{\prime}\right\}\right.
\end{aligned}
$$

Assumption I: $\Phi_{n}$ is a slowly varying function of $z, \partial \phi_{n^{\prime}} / z_{I_{-}^{2}} \div 0$.

Thus

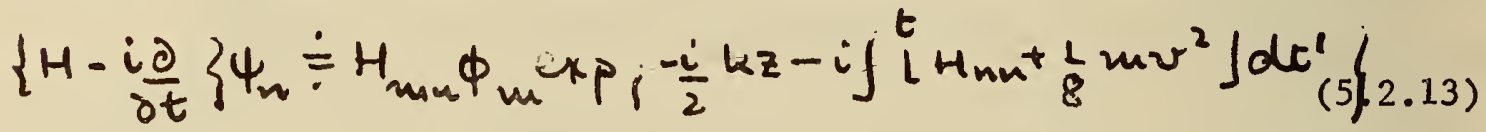

$$
\begin{aligned}
& \text { Using this, (5.2.11) becomes } \\
& \left.C_{n}{ }^{H} \text { mn } \Phi_{m} \exp 1-i \frac{k z}{2}-i\right\rfloor^{t}\left\lfloor H_{n n}+\frac{1}{8} m v^{2}\right\rfloor d t^{\prime} \text {; } \\
& \left.\left.+i_{m} H_{n m} \phi_{n} \exp \left\{t \frac{i k z}{2}-i\right\}^{t} L H_{m m}+\frac{1}{8} m v^{2}\right] d t^{\prime}\right\} \\
& =i \dot{c}_{n} \psi_{n}+i i_{m} \psi_{m}
\end{aligned}
$$

Multiply in turn by $\Psi_{m}^{*}, \Psi_{n}^{*}$ on the left and integrate over the electron co-ordinates, to obtain 


$$
\begin{aligned}
i \dot{i}_{m}= & i_{n} H_{m n} \exp \left\{-i j\left[H_{m n}-H_{m m}\right] d t^{\prime}\right\} \\
& +c_{m} H_{n m} \int \phi_{m}^{*} \phi_{n} e^{i k z} d r
\end{aligned}
$$

$$
\begin{aligned}
i \dot{C}_{n} & =i_{n} H_{n m} \exp \left\{-i \int^{t}\left[H_{m m}-H_{n n}\right] d t^{\prime}\right\} \\
& +c_{n} H_{\operatorname{mn}} \int \phi_{n}^{*} \phi_{m} e^{i k z} d r
\end{aligned}
$$

and make

Assumption II: $\quad e^{i k z} \div 1,(v \rightarrow 0)$.

Then

$$
\begin{aligned}
& i C_{m}^{0}=C_{n} H_{m u} \exp \left\{-i \int^{t}\left[H_{n n}-H_{m m}\right] d t^{\prime}\right\} \\
& i C_{n}^{0}=i_{m} H_{n m x^{2} x p}\left\{+i \int^{t}\left[H_{n n}-H_{m m} / d t^{\prime}\right\}\right. \\
& \text { It is true that near } R_{x} \\
& \frac{\partial H_{m n}}{\partial t}=0
\end{aligned}
$$

and thus in this region $(t \ll 1)$ a power series expansion gives, $\left(t=0\right.$ at $\left.R=R_{x}\right)$,

$$
\left(\mathrm{H}_{\mathrm{mm}}-\mathrm{H}_{\mathrm{nn}}\right) \div \alpha t
$$


where $\alpha$ is a constant. We now make

Assumption III: Equation $(5.2 .18)$ is valid for all $t$.

We can now write $(5.2 .16)$ as

$i C_{m}=C_{n} H_{m n} e^{-\frac{i}{2} \alpha t}$

$i \dot{c}_{n}=c_{m} H_{n m} e^{t \frac{i}{2} \alpha t}$

and eliminate $\mathrm{C}_{\mathrm{n}}$ to obtain $\left(\mathrm{f}=\left|\mathrm{H}_{\mathrm{mn}}\right|\right)$.

$\dot{c}_{m}+i \alpha t i_{m}+f^{2} i_{m}=0$

This is a version of Weber's equation, and solving it by standard methods subject to the boundary conditions

$$
C_{n}(-\infty)=1 \quad C_{m}(-\infty)=0
$$

we obtain

$$
\left|c_{m}(+\infty)\right|^{2}=1-e^{-w}
$$

with

$$
\omega=2 \bar{u}\left|H_{\text {nm }}\right|^{2} \frac{\partial}{\partial t}\left|H_{\text {min }}-H_{\text {un }}\right|_{R_{x}}
$$

yielding

$$
P=e^{-w}
$$

This is the Landau - Zener result. We now examine its validity. Taking the simplest problem first we have assumption II, that $\mathrm{e}^{i k z} \div 1$ as $\mathrm{v} \rightarrow 0$. This will be sufficiently accurate if 
$\sin (k z)<\frac{1}{2}$, or $k z<\frac{\pi}{4}$ say, so that if $\ell$ is a typical linear dimension of either of the atomic orbitals involved, we will have assumption II valid if the impact energy $\epsilon_{i}<\epsilon_{10}$ where ${ }^{(14)}$

$$
\varepsilon_{10} \sim \frac{2.5 \times 10^{4} \mathrm{M}}{\ell^{2}} \mathrm{eV}
$$

where $M$ is in a.m.u. and $\ell$ in atomic units. Clearly this is not a very severe restriction. Assumptions 0 and $I$ are much more significant. They imply that both $\phi_{m}$ and $\phi_{n}$ describe $s$ states, for if say $\phi_{n}$ is not, then $\partial \phi_{n} / \partial z_{\text {I }}$ contains appreciable components of states differing from $\phi_{\mathrm{n}}$ in azimuthal quantum number alone. For example, Bates (1962) points out that if $\phi_{m}$ is a $p_{0}$ state an $S$ state transitions of the sequence $\mathrm{S} \rightarrow \mathrm{p}_{\mathrm{o}} \rightarrow \mathrm{p}_{ \pm 1} \rightarrow \mathrm{P}_{\mathrm{O}} \rightarrow \mathrm{S}$ will considerably decrease the Landau - Zener $\mathbf{P}$ factor. Even if the states are both s type, at sufficiently high velocity the assumption $\partial \Phi_{n} / \partial z_{r_{1}} \sim 0$ must be false. The most serious failure, however, was pointed out by Bates ${ }^{(14)}$ and this is the falsity of assumption III. He points out that transitions are in fact likely throughout the region in which the exponential factors in (5.2.16) are slowly varying. If the width of this zone is $\Delta z$, then we have, by the method of stationary phase,

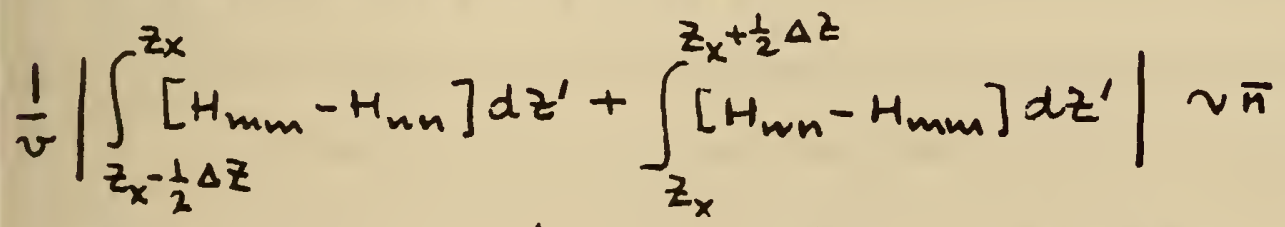

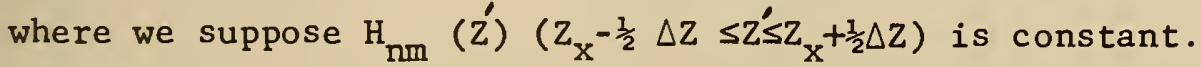


Making assumption III,

$$
\frac{1}{v}\left|\int_{z_{x}-\frac{1}{2}}^{z_{x}} \alpha z d z-\int_{z_{x}}^{z_{x}+\frac{1}{2} \Delta z} \alpha z d z\right| \sim \bar{u}
$$

or

$$
\Delta z \sim\left(\frac{4 \pi v}{\alpha}\right)^{1 / 2}
$$

That is, $\Delta z \rightarrow \infty$ as $v \rightarrow \infty$ and this is clearly unacceptable; so III cannot be valid. Even when $\mathrm{v}$ is small $\Delta \mathrm{Z}$ is so large that it is not in general sufficient to treat $H_{n m}$ as constant. When $P$ is a maximum $(\omega=0.69)$, Bates shows that

$$
\Delta \mathrm{z} \div 0.5\left|\mathrm{H}_{\mathrm{nm}}\left(\mathrm{R}_{\mathrm{x}}\right)\right| \mathrm{z}_{\mathrm{x}}^{2}
$$

with $\mathrm{H}_{\mathrm{nm}}$ in ev., which may be large. The correct procedure in the simple case of $s$ - $s$ transitions, is to solve $(5.2 .16)$ as it stands to obtain $\left(\mathrm{C}_{\mathrm{m}} \ll \mathrm{C}_{\mathrm{n}}\right)$

$P=2\left|\frac{1}{v} \int_{-\infty}^{+\infty} H_{n m} e x_{0}\left\{-\frac{i}{v} \int_{0}^{z}\left[H_{n n}-H_{m m}\right] d z^{\prime}\right\} d z\right|^{2}$

which is very different from $(5 \cdot 2.23)$. This has not as yet been applied to any specific case.

To sum up, one concludes that the Landau - Zener formulation may be satisfactory for S-S transitions when $\mathrm{v}$ is well below the velocity $U_{m}$ at which the maximum cross section is expected. 
REFERENCES

1. Brinkmann and Kramers (1930), Proc. Acad. Sci. Amsterdam 33, 973.

3. Oppenheimer, J. R., (1928), Phys. Rev. 31, 349.

4. Bates and Dalgarno, (1952), Proc. Phys. Soc. 뜨, 919.

5. Barnett and Reynolds, (1958), Phys. Rev. 109, 355.

6. Bates and Dalgarno, (1953), Proc. Phys. Soc. 스느, 972.

7. Jackson and Schiff, (1953), Phys. Rev. 89, 359.

8. McDowell, (1961), Proc. Roy. Soc. A, Vol. 264, 277.

9. Bassel and Gerjnoy, (1960), Phys. Rev. 117, 749.

11. McDowell, (1962), To be published.

12. Landau, (1932), Phys. Z. Sowjetunion 2, 46 .

13. Zener, C., (1932), Proc. Roy. Soc. A 137, 696.

14. Bates, D. R., (1960), Proc. Roy. Soc. A 257, 22. 


\section{LECTURE VI}

\section{Ionization of Atomic Systems By Fast Protons}

This final lecture will describe what little work has been done up to the present on the ionization of atomic systems by other atomic systems, which are usually supposed bare ions.

Following (2.13) we have for the transition

$$
\mathrm{X}^{+}+\mathrm{Y} \rightarrow \mathrm{X}^{+}+\mathrm{Y}^{+}+\mathrm{e}
$$

the electron being ejected with momentum $\underline{\mathrm{K}}$,

$$
Q_{k}(n l \rightarrow c)=\frac{1}{2 \pi v^{2}} \int_{K_{\min }}^{k_{\max }}\left|N_{n m}\right|^{2} K d k
$$

and

$$
Q_{\text {ion }}\left(v^{2}\right)=\int_{0}^{x_{\max }} Q_{12}(n l \rightarrow c) d \mathcal{R}
$$

where $\mathrm{N}_{\mathrm{nm}}(\mathrm{K}, \boldsymbol{H})$ is the relevant matrix element.

Apart from some external numerical factor depending on the atomic states involved, this will in general be of the form,

$$
N(u e \rightarrow c)=\int \underline{\psi}_{i}\left(\underline{r}_{j}\right) \sum_{j} e^{i \underline{k} \cdot \tau_{j}} \bar{\psi}_{f}\left(\underline{r}_{j}\right) d \underline{r}_{j}
$$

where we are now working to Born's approximation. Now $\Psi_{f}\left(\underline{r}_{j}\right)$ is of the form,

$$
\Psi_{f} \sim P\left[u\left(x_{1}, r_{2}, \ldots, r_{j-1}, r_{j+1}, \cdots, I_{n}\right) \frac{\mathcal{R}}{(2 n)^{3 / 2}} F\left(r_{j}\right)\right]_{(6.5)}
$$

where $P$ permutes all the $\underline{r}_{1}(i \neq j)$ with $\underline{r}_{j}$, and the $j$ th electron is ejected, and $U$ is the wave function of the residual ion. Here we have normalized the ejected electron wave function 
$\psi_{\underline{x}}\left(\underline{r}_{j}\right)$ such that

$\int \Psi_{x^{*}}^{*}\left(\underline{r}_{j}\right) \underline{\Psi}_{x^{\prime}}\left(\underline{r}_{j}\right) d \underline{r}_{j}=\delta\left(\underline{\alpha}^{\prime}-\underline{\not}\right)$

so that $F\left(\underline{r}_{\jmath}\right)$ has asymptotic form

$$
F\left(\underline{r}_{j}\right) \sim e^{i \sigma(x)+i \underline{\underline{R} \cdot r}}
$$

where $\sigma$ is a phase factor.

Integrating over $\underline{R}$ and all the electrons except the $j$ th, the integral (6.4) turns out to be proportional to

$$
I(n e \rightarrow c)=\int \phi\left(n e, r_{j}\right) \phi\left(x, r_{j}\right) e^{i \underline{k} \cdot \underline{r}_{j}} d \underline{r}_{j}
$$

Two alternative procedures are possible. If we are willing to suppose that the ejected electron moves in a Coulomb field, of some effective charge $\mathrm{Z}$, we can write

$$
\phi\left(x, z, r_{j}\right)=N\left(\gamma^{2}\right) e^{i \bar{u} z / 2} e^{i \alpha r}
$$

and perform the integration by standard methods, provided

$\Phi\left(n, \underline{r}_{j}\right)$ has a reasonably simple analytic form. For hydrogen $(z=1)$ this is of course exact, and the calculations for both $\mathrm{H}^{\circ}$ and $\mathrm{H}^{+}$impact have been performed by Bates and $\operatorname{Griffing}(1,2)$ and are shown in Fig. (6.1).

The alternative procedure is to expand $\Phi\left(x, \underline{r}_{j}\right)$ in partial waves,

$\phi\left(2, r_{j}\right)=\sum_{l}(2 l+1) i^{l} e^{i \sigma_{q}} R_{2 l l}(r) P_{l}(\cos \theta)$ 
58

where $R_{R e}(r)$. is the solution of the Schrodinger equation for an electron in the field of $\mathrm{Y}^{+}\left(\mathrm{n}^{\prime} ' \ell^{\prime \prime}\right)$, having an electron of nomentum $x$ in the $l^{\prime}$ wave of the continuum. The cross section then becomes

$$
Q_{\text {ion }}=\frac{\text { oust }}{S_{i}^{2}} \sum_{l^{\prime}}\left(\partial l^{\prime}+1\right) Q_{l^{\prime}}\left(S_{i}\right)
$$

with

$$
Q_{l^{\prime}}\left(S_{i}\right)=\int_{0}^{\lambda_{\text {max }}} G_{1 \ell^{\prime}}\left(S_{i}\right) \mathcal{K} d \mathcal{K}
$$

$$
\begin{aligned}
& G_{12, e^{\prime}}\left(S_{i}\right)=\int_{12 \text { min }}^{1+\max }\left|G_{12, e^{\prime}}(K)\right|^{2} K^{-3} d K \\
& G_{1, e^{\prime}}(K)=\int P(u e, r) P\left(\left\{r e^{\prime}, r\right) \dot{\gamma}_{e}(K r) d r\right.
\end{aligned}
$$

Here $P(n \ell, r)=r R(n \ell, r)$, and $j_{l}$ is the usual $\ell$ th order spherical Bessel function.

ss. We now discuss the application of these procedures to atoms other than hydrogen.

(i) Helium. This has been investigated by Erskine( ${ }^{(3)}$, Dalgarno and McDowell ${ }^{(4)}$, Mapleton ${ }^{(5)}$, and Grosjean and V. d Wale ${ }^{(6)}$. All the above authors represent the ground 1 s state by a simple one parameter variational wave function

$$
\phi(1 s, r)=\frac{z_{1}^{3 / 2}}{\sqrt{\pi}} e^{-z_{1} r} \quad ; z_{1}=1.69
$$


Mapleton takes

$\phi\left(z l^{\prime}, r\right)=N(k) e^{i x r} F\left[-\frac{i z_{2}}{k}, 1, i k k_{r}-i \underline{z} \cdot r\right]$

With this choice one must take $z_{2}=z_{1}$ to ensure orthogonality, otherwise one obtains a cross section tending to a finite limit as $E_{1} \rightarrow \infty$. (Fig. 6.1). However, asymptotically one has a Coulomb field with $Z_{2}=1.0$. A better choice therefore is to expand (6.16) in partial waves and take

$$
\begin{array}{ll}
z_{2}=z_{1} & l=0 \\
z_{2}=1 & (l \neq 0)
\end{array}
$$

His results for this case are in excellent agreement with experiment $(7,8)$ (Fig. 6.2) and with the calculations of Erskine, who ignored the $\ell=0$ contribution and took $P(x, l=1, r)$ to be the solution of the Hartree equation

$\frac{d^{2} P(k \ell, r)}{d r^{2}}+\left\{k^{2}+\frac{2}{r}\left[1+(1+2 r) e^{-4 r}\right]-\frac{l(l+1)}{r^{2}}\right\} P(k \ell, r)=0$

as did Grosjean and V. d Waale. Dalgarno and McDowell solved

(6.18) with a numerical potential term, and used this to obtain $z_{2}(x)$ for the $p$ - wave, evaluating the other contributions with the same $z_{z}(x)$.

(ii) Lithium. McDowell and Peach ${ }^{(13)}$ pointed out that in this case their is no choice of $z_{2}$ which will ensure orthogonality, and that the cross section with Coulomb waves diverges for all $z_{2}$. However, the dominant $p$ - wave contribution is finite and was 
computed for $Z_{2}=Z_{1}$ and $Z_{2}=1$, (Fig. 6.3), and is clearly very sensitive to the choice of $z_{2}$. They show that the corresponding photoionization cross section varies as $Z_{2}{ }^{7} \exp \left\{-\beta Z_{2}\right\}$ Even for $Z_{2}=1, Q_{\text {photo }}$ is sensitive to the choice of bound state wave function. (Table 6.1)

\section{TABLE 6.1}

Photoionization cross sections of $\mathrm{Li}\left(10^{-18} \mathrm{~cm}^{2}\right)$ with Coulomb function $Z=1$ and (a) 3 - parameter bound function (Holoien 1958) ${ }^{(9)}$

(b) 9 - parameter bound function (Roothan et al., 1960) ${ }^{(10)}$

$\begin{array}{lccc}x^{2} \text { (Rydbergs) } & \mathrm{a} & \mathrm{b} & \mathrm{c} \\ 0 & 0.769 & 0.513 & 2.50 \\ 0.01 & 0.819 & 0.547 & -- \\ 0.02 & 0.869 & 0.634 & 2.40 \\ 0.03 & 0.915 & 0.726 & --- \\ 0.04 & 0.956 & 0.779 & 2.20 \\ 0.05 & 0.982 & 0.772 & --\end{array}$

"c" indicates the experimental results of Tunstead (1953).

This implies that the agreement between Mapleton's calculations and the experimental results may well be fortuitous.

McDowe 11 and Peach are now recomputing $Q_{i o n}$ for $\mathrm{H}^{+}$on $\mathrm{Li}$ using Hartree - Fock wave functions for the ejected electron and a 3-parameter Holoien function for the bound state. 


\section{(iii) Other Calculations.}

bates and Griffing $(1,2)$, McDowell and Peach $(12,13)$, and Bates, McDowell and Omholt ${ }^{(14)}$ have calculates the energy distribution of the ejected electrons at energy $E_{1}$, from $H, H^{-}, \mathrm{Li}$ and $\mathrm{Ne}$ respectively for either $\mathrm{H}^{+}$or $\mathrm{H}^{\rho}$ impact. They find it depends primarily on the initial angular momentum $l$, as would be expected from consideration of the photoionization cross sections. Rudd has extended this work to He using Mapleton's calculations, and finds reasonable agreement with experiment.(8) (Fig. 6.4).

\section{(iv) Classical Calculations.}

Rudd (loc. cit.) and Alsmiller(15) have applied Grsinskis' modification of Thompson's method to calculate $Q_{\text {ion }}$ for $\mathrm{H}^{+}$on He and $\mathrm{H}_{2}$ respectively. (Fig. 6.2 and 6.4 ). They obtain surprisingly good agreement with the observations, considering the nature of the approximation. [In this method one assumes that if the ion passes at impact parameter $p$ it will transfer energy $\Delta E(p, v)$ to an electron of velocity $\mathrm{v}$, and computes the cross section as

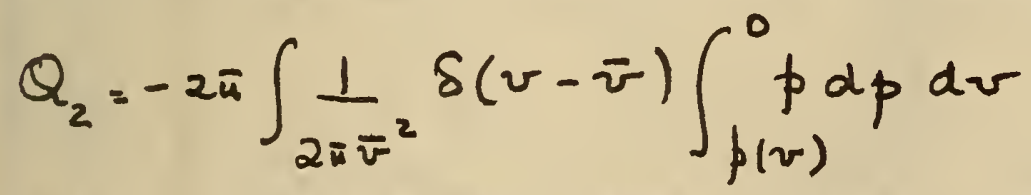

where $\bar{v}$ is the expectation value of the velocity and $p(v)$ is the greatest impact parameter for which $\Delta E(p, v)$ exceeds the ionization potential.] 


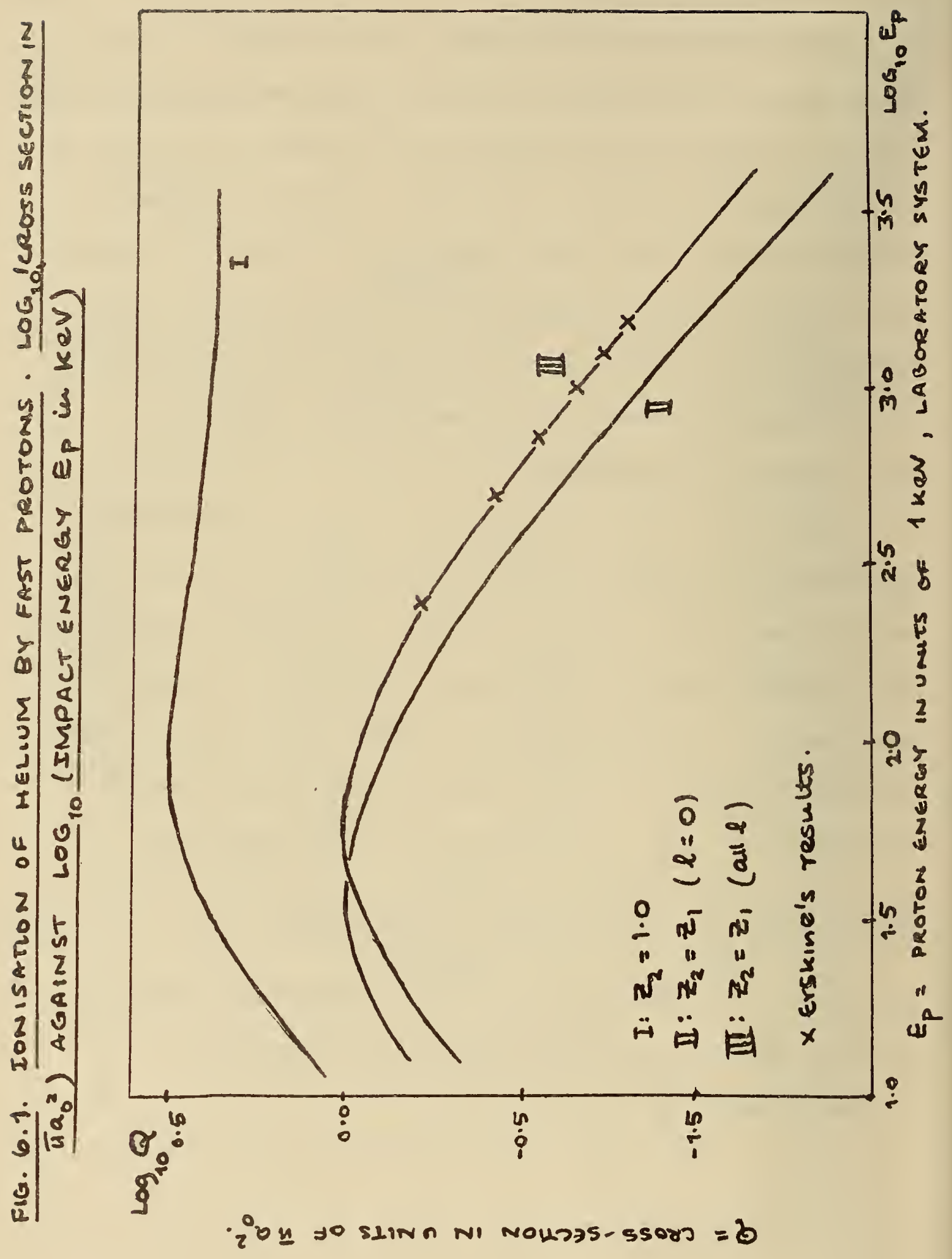




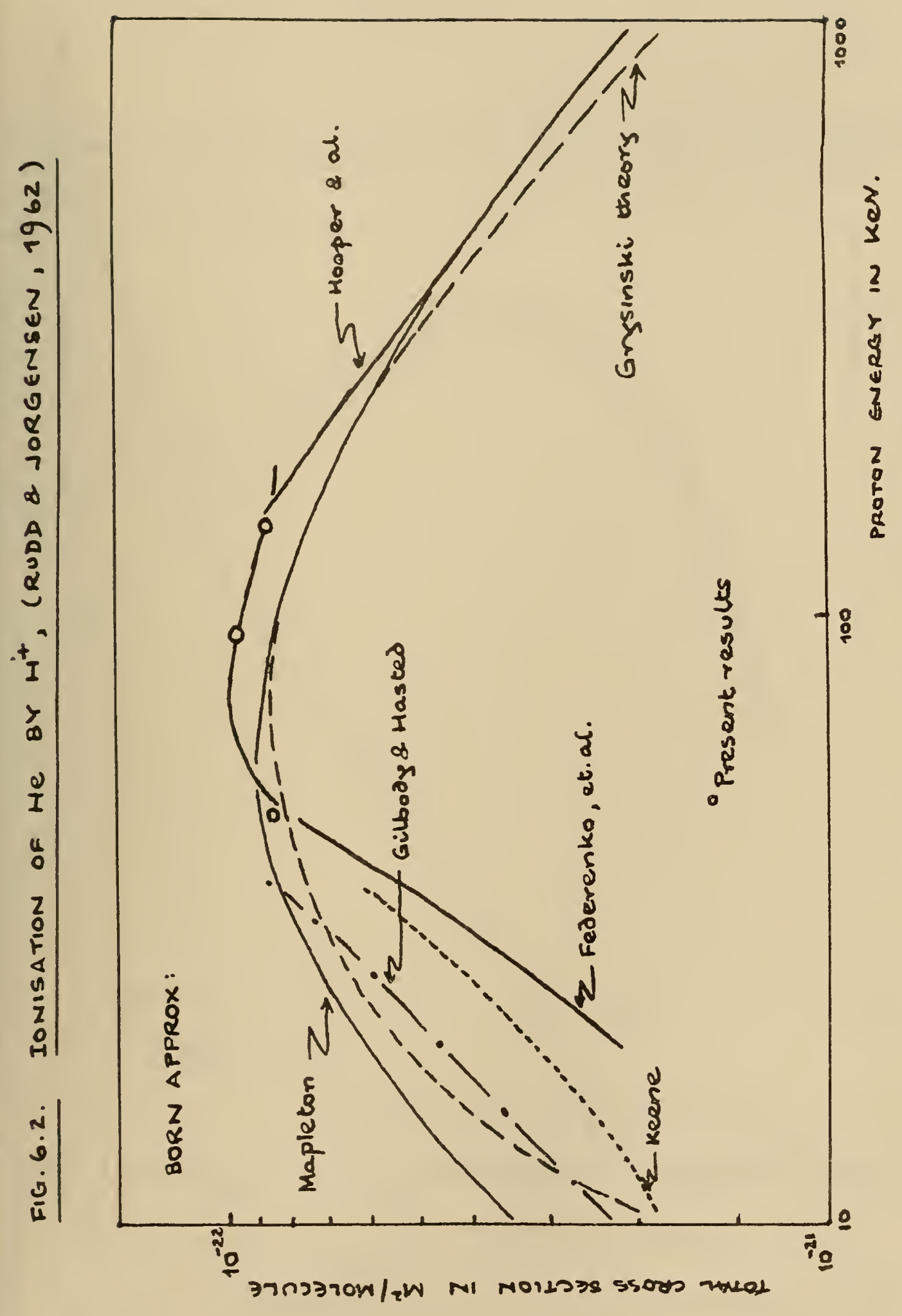


FIG. 6.3. IONISATION OF LITHIUM BY PROTON IMPACT.

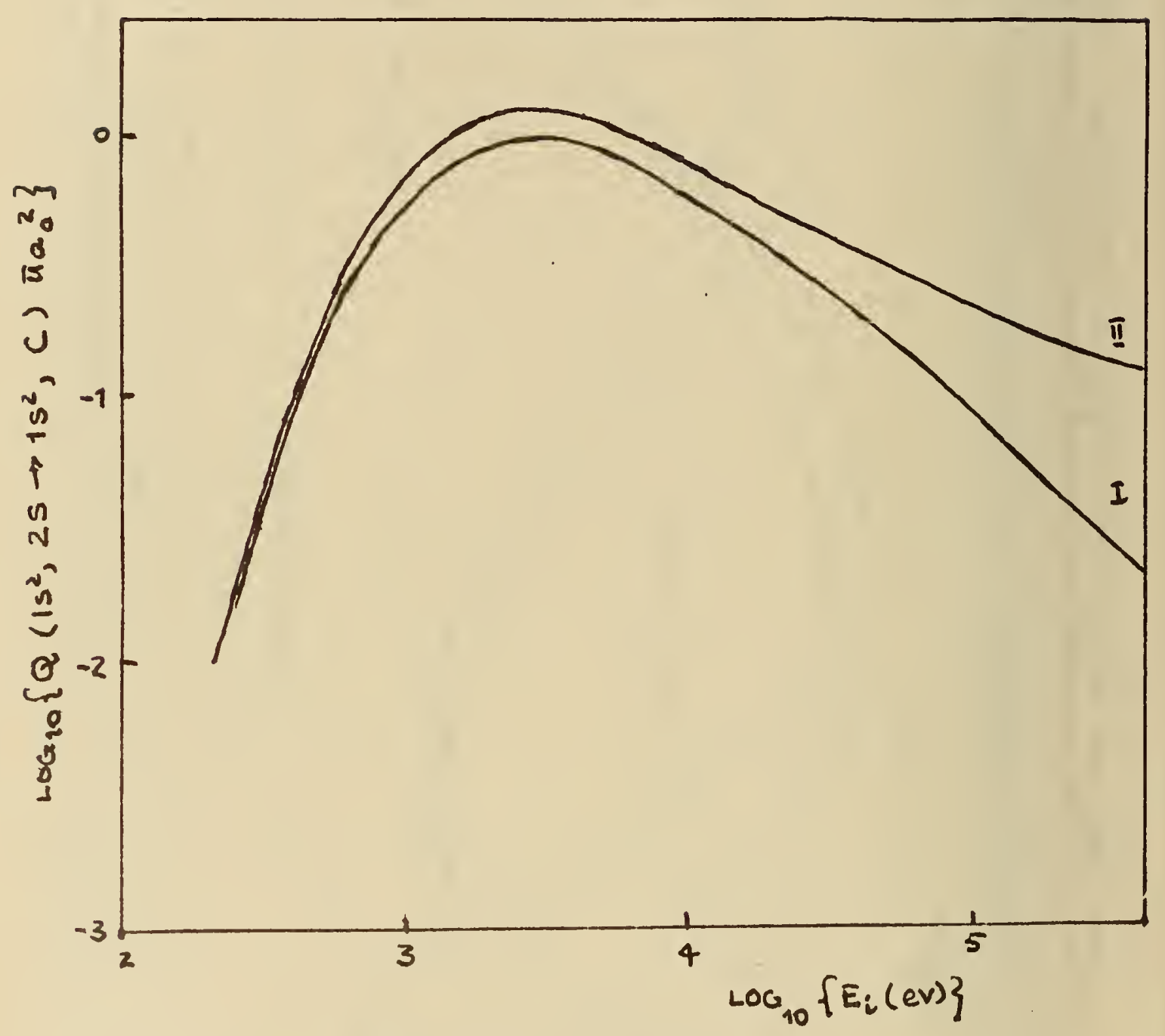

I: p.w wave Born $z_{1}=z_{2}=2 \cdot 67$.

II: Total cross-section $Z_{1}=Z_{2}$ 


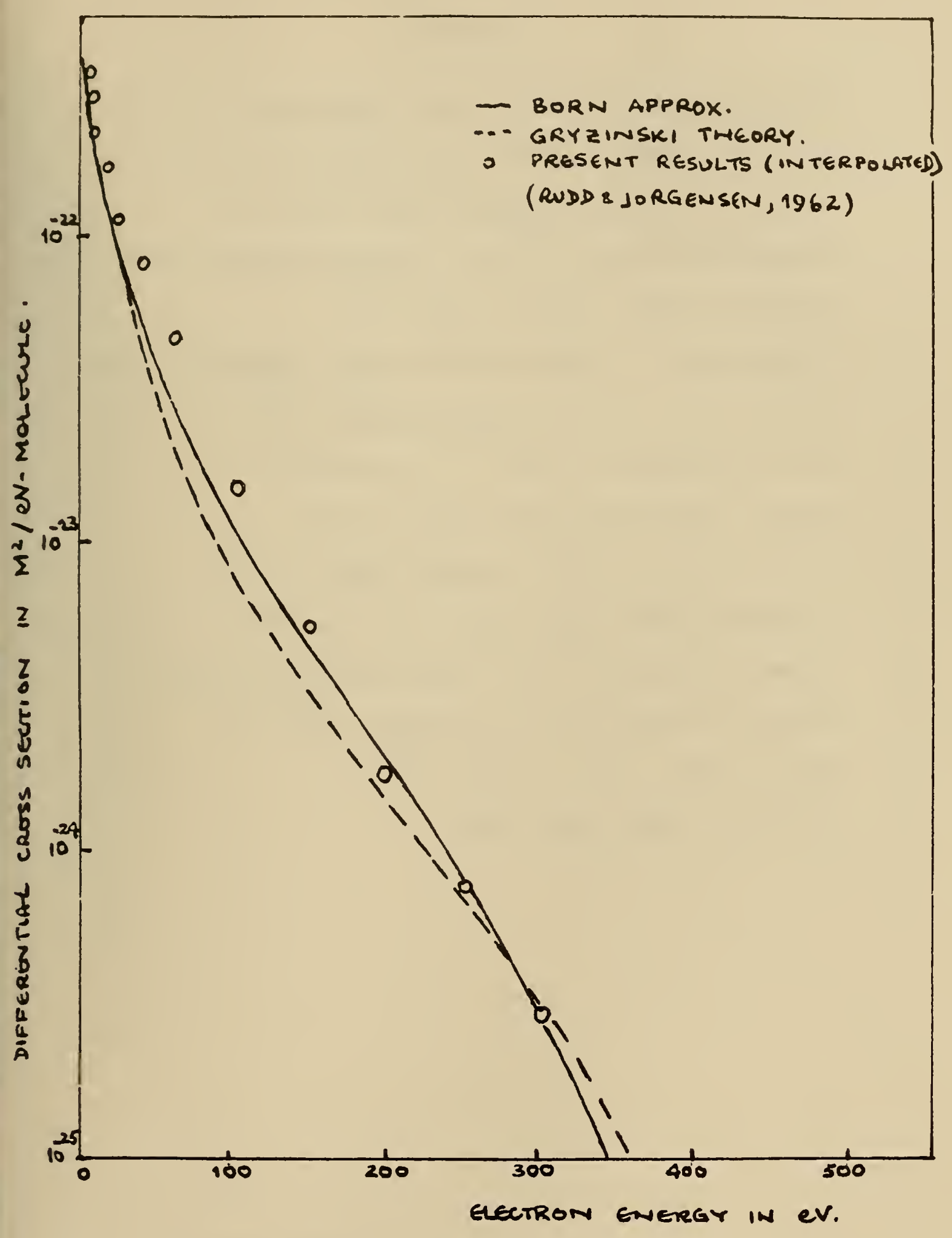

FIG.6.4. IONISATION OF HE BY H'; ELECTRON DIST: 


\section{REFERENCES}

1. Bates and Griffing, (1953), Proc. Phys. Soc. ‥ 66, 961.

2. Bates and Griffing, (1954), Proc. Phys. Soc. A, 68, 90.

3. Erskine, G., (1954), Proc. Roy. Soc. A, 224, 362.

4. Dalgarno and McDowel1, (1956), Airglow and Auroae. (1oc. cit.).

5. Mapleton, (1958), Phys. Rev. 109, 1166.

6. Grosjean and V. d. Waale, (1961), Nuovo. Ginento. 19, 232.

7. Hooper, et al., (1961), Phys. Rev. 121, 1123.

8. Rudd and Jorgenson, (1962), To be published.

9. Holoien, (1958), Proc. Phys. Soc. 72, 461.

10. Roothan, et al., (1960), Rev. Mod. Phys. 33, 221.

11. Tunstead, (1953), Proc. Phys. Soc. A, 66, 304.

12. McDowe11 and Peach, (1959), Proc. Phys. Soc. 74, 463.

13. McDowe11 and Peach, (1961), Phys. Rev. 121, 1383.

14. Bates, etª1., (1957), J.A.T.P. 10, 51 .

15. A1smiller, (1961), Priv. Comm. 


Luther II. llodges, Secretary

VATIONAL BUREAU OF STANDARDS

A. V. Astin, Director

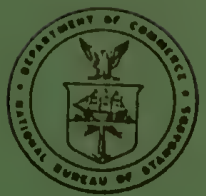

\section{THE NATIONAL BUREAU OF STANDARDS}

The scope of activities of the National Bureau of Standards at its major laboratories in Washington, D.C., and Boulder, Colorado, is suggested in the following listing of the divisions and sectionsengaged in technical work. In general, each section carries out specialized research, development, and engineering in the field indicated by ils title. A brief description of the activities, and of the resultant publications, appears on the inside of the front cover.

A ISHINGTON, D. C.

Electricity. Resistance and Reactance. Electrochemistry. Electrical Instruments. Magnetic Measurements Pielectrics. High Voltage.

Metrology. Photometry and Colorimetry. Refractometry. Photographic Research. Length. Engineering Metrology. Mlass and Scale. Volumetry and Densimetry.

Heat. Temperature Physics. Heat Measurements. Cryogenic Physics. Equation of State. Statistical Physics. Radiation Physics. X-ray. Radioactivity. Radiation Theory. High Energy Radiation. Radiological Equipment. Xucleonic Instrumentation. Neutron Physics.

Analytical and Inorganic Chemistry. Prure Substances. Spectrochemistry. Solution Chemistry. Standard Reference Materials. Applied Analytical Research. Crystal Chemistry.

Mechanics. Sound. Pressure and Vacuum. Fluid Mechanics. Engineering Mechanics. Rheology. Combustion Controls.

Polymers. Macromolecules: Synthesis and Structure. Polymer Chemistry. Polymer Physic s. Polymer Characterization. Polymer Evaluation and Testing. Applied Polymer Standards and Research. Dental Research.

Metallurgy. Engineering Metallurgy. Microscopy and Diffraction. Metal Reactions. Metal Physics. Electrolysis and Metal Deposition.

Inorganic Solids. Engineering Ceramics. Glass. Solid State Chemistry. Crystal Growth. Physical Properties. Crystallography.

Building Research, Structural Engineering. Fire Research. Mechanical Systems. Organic Building Materials. Codes and Safety Standards. Heat Transfer. Inorganic Building Materials. Metallic Building Materials.

Applied Mathematics. Numerical Analysis. Computation. Statistical Engineering. Mathematical Physics. Operations Research.

Data Processing Systems. Components and Techniques. Computer Technology. Measurements Automation. Engineering Applic ations. Systems Analysis.

Atomic Physics. Spectroscopy. Infrared Spectroscopy. Far Ultraviolet Physics. Solid State Physics. Electron Physics. Atomic Physics. Plasma Spectroscopy.

Instrumentation. Engineering Electronics. Electron Devices. Electronic Instrumentation. Mechanical Instruments. Basic lnstrumentation.

Physical Chemistry. Thermochemistry. Surface Chemistry. Organic Chemistry. Molecular Spectroscopy. Elementary Processes. Mass Spectrometry. Photochemistry and Radiation Chemistry.

ofrice of Weights and Measures.

BOULDER, COLO.

Cryogenic Engineering Laboratory. Cryogenic Equipment. Cryogenic Processes. Properties of Materials. Cryogenic Technical Services.

\section{CENTRAL, RADIO PROPAGATION LABORATORY}

Ionosphere Research and Propagation. Low Frequency and Very Low Frequency Research. lonosphere Research. Prediction Services. Sun-Earth Relationships. Field Engineering. Radio Warning Services. Vertical Soundings Research.

Radio Propagation Engineering. Data Reduction Instrumentation. Radio Noise. Tropospheric Measurements. Tropospheric Analysis. Propagation-Terrain Effects. Radio-Meteorology. Lower Atmosphere Physic s.

Radio Systems. Applied F.lectromagnetic Theory. High Frequency and Very High Frequency Research. Frequency Utilization. Modulation Research. Antenna Research. Radiodetermination.

Upper Atmosphere and Space Physics. Upper Atmosphere and Plasma Physics. High Latitude Ionosphere Physics. lonosphere and Exosphere Scatter. Airglow and Aurora. Ionospheric Radio Astronomy.

\section{RADIO STANDARDS LABORATORY}

Radio Physics. Radio Broadcast Service. Radio and Microwave Materials. Atomic Frequency and Time-Interval Standards. Radio Plasma. Millimeter-Wave Research.

Circuit Standards. High Frequency Electrical Standards. High Frequency Calibration Services. High Frequency Impedance Standards. Microwave Calibration Services. Microwave Circuit Standards. Low Frequency Calibration Services. 
